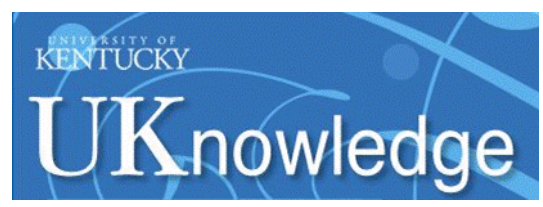

University of Kentucky

UKnowledge

$12-18-2018$

\title{
Detecting Radio AGN Signatures in Red Geysers
}

\author{
Namrata Roy \\ University of California - Santa Cruz \\ Kevin Bundy \\ University of California - Santa Cruz \\ Wiphu Rujopakarn \\ The University of Tokyo, Japan \\ Michele Cappellari \\ University of Oxford, UK \\ Francesco Belfiore \\ University of California - Santa Cruz
}

See next page for additional authors

Follow this and additional works at: https://uknowledge.uky.edu/physastron_facpub

Part of the Astrophysics and Astronomy Commons

Right click to open a feedback form in a new tab to let us know how this document benefits you.

\section{Repository Citation}

Roy, Namrata; Bundy, Kevin; Rujopakarn, Wiphu; Cappellari, Michele; Belfiore, Francesco; Yan, Renbin; Heckman, Tim; Bershady, Matthew; Greene, Jenny; Westfall, Kyle; Drory, Niv; Rubin, Kate; Law, David; Zhang, Kai; Gelfand, Joseph; Bizyaev, Dmitry; Wake, David; Masters, Karen; Thomas, Daniel; Li, Cheng; and Riffel, Rogemar A., "Detecting Radio AGN Signatures in Red Geysers" (2018). Physics and Astronomy Faculty Publications. 609.

https://uknowledge.uky.edu/physastron_facpub/609

This Article is brought to you for free and open access by the Physics and Astronomy at UKnowledge. It has been accepted for inclusion in Physics and Astronomy Faculty Publications by an authorized administrator of UKnowledge. For more information, please contact UKnowledge@lsv.uky.edu. 
Detecting Radio AGN Signatures in Red Geysers

Digital Object Identifier (DOI)

https://doi.org/10.3847/1538-4357/aaee72

Notes/Citation Information

Published in The Astrophysical Journal, v. 869, no. 2, 117, p. 1-14.

(c) 2018. The American Astronomical Society. All rights reserved.

The copyright holder has granted the permission for posting the article here.

Authors

Namrata Roy, Kevin Bundy, Wiphu Rujopakarn, Michele Cappellari, Francesco Belfiore, Renbin Yan, Tim Heckman, Matthew Bershady, Jenny Greene, Kyle Westfall, Niv Drory, Kate Rubin, David Law, Kai Zhang, Joseph Gelfand, Dmitry Bizyaev, David Wake, Karen Masters, Daniel Thomas, Cheng Li, and Rogemar A. Riffel 


\title{
Detecting Radio AGN Signatures in Red Geysers
}

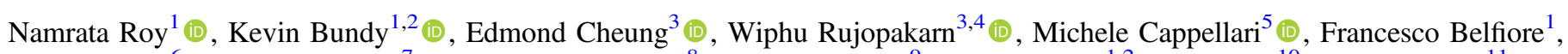

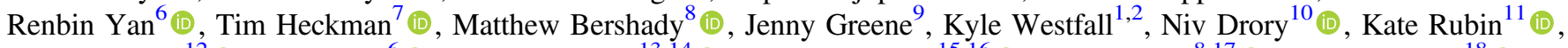 \\ David Law $^{12}$ (D), Kai Zhang ${ }^{6}$ (D), Joseph Gelfand ${ }^{13,14}$ (D), Dmitry Bizyaev ${ }^{15,16}$ (iD), David Wake ${ }^{8,17}$ (iD), Karen Masters ${ }^{18}$ (D), \\ Daniel Thomas ${ }^{18}$, Cheng Li ${ }^{19}$, and Rogemar A. Riffel ${ }^{20,21}$ \\ ${ }^{1}$ Department of Astronomy and Astrophysics, University of California, 1156 High Street, Santa Cruz, CA 95064, USA; naroy@ucsc.edu \\ ${ }^{2}$ UCO/Lick Observatory, Department of Astronomy and Astrophysics, University of California, 1156 High Street, Santa Cruz, CA 95064, USA \\ ${ }^{3}$ Kavli Institute for the Physics and Mathematics of the Universe (WPI), The University of Tokyo Institutes for Advanced Study, \\ The University of Tokyo, Kashiwa, Chiba 277-8583, Japan \\ ${ }^{4}$ Department of Physics, Faculty of Science, Chulalongkorn University, 254 Phayathai Road, Pathumwan, Bangkok 10330, Thailand \\ ${ }^{5}$ Sub-department of Astrophysics, Department of Physics, University of Oxford, Denys Wilkinson Building, Keble Road, Oxford OX1 3RH, UK \\ ${ }^{6}$ Department of Physics and Astronomy, University of Kentucky, 505 Rose Street, Lexington, KY 40506-0055, USA \\ ${ }^{7}$ Center for Astrophysical Sciences, Department of Physics \& Astronomy, The Johns Hopkins University, Baltimore, MD 21218, USA \\ ${ }^{8}$ Department of Astronomy, University of Wisconsin-Madison, 475 North Charter Street, Madison, WI 53706, USA \\ ${ }^{9}$ Department of Astrophysical Sciences, Princeton University, Princeton, NJ 08544, USA \\ ${ }^{10}$ McDonald Observatory, Department of Astronomy, University of Texas at Austin, 1 University Station, Austin, TX 78712-0259, USA \\ ${ }^{11}$ Harvard-Smithsonian Center for Astrophysics, 60 Garden Street, Cambridge, MA 02138, USA \\ ${ }^{12}$ Space Telescope Science Institute, 3700 San Martin Drive, Baltimore, MD 21218, USA \\ ${ }^{13}$ NYU Abu Dhabi, P.O. Box 129188, Abu Dhabi, UAE \\ ${ }^{14}$ Center for Cosmology and Particle Physics, New York University, Meyer Hall of Physics, 4 Washington Place, New York, NY 10003, USA \\ ${ }^{15}$ Apache Point Observatory and New Mexico State University, P.O. Box 59, Sunspot, NM 88349-0059, USA \\ ${ }^{16}$ Sternberg Astronomical Institute, Moscow State University, Moscow, Russia \\ ${ }^{17}$ Department of Physical Sciences, The Open University, Milton Keynes, MK7 6AA, UK \\ ${ }^{18}$ Institute for Cosmology and Gravitation, University of Portsmouth, Dennis Sciama Building, Burnaby Road, Portsmouth PO1 3FX, UK \\ ${ }^{19}$ Tsinghua Center for Astrophysics \& Physics Department, Tsinghua University, Beijing 100084, China \\ ${ }^{20}$ Universidade Federal de Santa Maria, Departamento de Física, CCNE, 97105-900, Santa Maria, RS, Brazil \\ ${ }^{21}$ Laboratório Interinstitucional de e-Astronomia-LIneA, Rio de Janeiro, RJ, Brazil \\ Received 2018 July 11; revised 2018 October 30; accepted 2018 November 2; published 2018 December 18
}

\begin{abstract}
A new class of quiescent galaxies harboring possible AGN-driven winds has been discovered using spatially resolved optical spectroscopy from the ongoing SDSS-IV MaNGA survey. These galaxies, termed "red geysers," constitute $5 \%-10 \%$ of the local quiescent population and are characterized by narrow bisymmetric patterns in ionized gas emission features. Cheung et al. argued that these galaxies host large-scale AGN-driven winds that may play a role in suppressing star formation at late times. In this work, we test the hypothesis that AGN activity is ultimately responsible for the red geyser phenomenon. We compare the nuclear radio activity of the red geysers to a matched control sample with similar stellar mass, redshift, rest-frame NUV $-r$ color, axis ratio, and presence of ionized gas. We have used the $1.4 \mathrm{GHz}$ radio continuum data from the VLA FIRST survey to stack the radio flux from the red geyser and control samples. In addition to a three times higher FIRST detection rate, we find that red geysers have a $5 \sigma$ higher level of average radio flux than control galaxies. After restricting to rest-frame NUV $-r$ color $>5$ and checking mid-IR WISE photometry, we rule out star formation contamination and conclude that red geysers are associated with more active AGNs. Red geysers and a possibly related class with disturbed H $\alpha$ emission account for $40 \%$ of all radio-detected red galaxies with $\log \left(M_{\star} / M_{\odot}\right)<11$. Our results support a picture in which episodic AGN activity drives large-scale, relatively weak ionized winds that may provide a feedback mechanism for many early-type galaxies.
\end{abstract}

Key words: galaxies: evolution - galaxies: formation - galaxies: general

\section{Introduction}

The level of star formation in galaxies is known to be bimodal (Strateva et al. 2001; Blanton et al. 2003; Kauffmann et al. 2003), with star-forming galaxies often referred to as the "blue cloud" and galaxies without significant star formation falling under the "red-sequence" category. The latter is characterized by old stellar populations ( $\geq 6 \mathrm{Gyr})$ and short star formation timescales ( $\lesssim 1 \mathrm{Gyr}$; Tinsley 1979; Worthey et al. 1992; Trager et al. 2000; Thomas et al. 2005; Graves \& Schiavon 2008; Choi et al. 2014; Conroy et al. 2014; Worthey et al. 2014). The abundance of these quiescent galaxies has increased by several factors since $z \sim 2$ (Bell et al. 2004; Bundy et al. 2006; Faber et al. 2007; Ilbert et al. 2010; Moustakas et al. 2013), which implies that more and more galaxies are transitioning to quiescence. The increase in the red-and-dead population indicates that once galaxies shut off their star formation by some mechanism, they must stay quenched for a long time.

A permanent shutdown of star formation is hard to explain, because the quiescent population is not devoid of gas and is also capable of accreting new gas to eventually start forming stars again. Major surveys have shown an abundance of gas in quiescent galaxies (Demoulin-Ulrich et al. 1984; Buson et al. 1993; Binette et al. 1994), which if left to itself, should ultimately cool and form stars. This gas comes from a variety of sources like stellar mass loss from evolved stars (e.g., Mathews \& Brighenti 2003; Ciotti \& Ostriker 2007) or minor mergers. If all this gas formed stars, we would expect the global 
stellar mass density to be larger by factors of a few than the observed at $z=0$. This implies that an additional feedback mechanism is required to maintain the suppression of star formation in galaxies on the red sequence (Benson et al. 2003).

While a number of feedback mechanisms have been proposed, including interstellar medium (ISM) heating from stellar winds (Conroy et al. 2015) and gravitational effects induced by galaxy bulges (Martig et al. 2009), the most popular explanation has been active galactic nucleus (AGN) feedback (Binney \& Tabor 1995; Ciotti \& Ostriker 2001; Croton et al. 2006; Fabian 2012; Heckman \& Best 2014; Yuan \& Narayan 2014). It states that the energy released from the central AGN in the host galaxy in the form of winds, outflows, or jets can significantly effect the evolution of the galaxy by a feedback mechanism, which can take place in two different ways (Fabian 2012; Morganti 2017). The "quasar" or "radiative" mode feedback, mostly associated with luminous AGNs or massive quasars, releases huge amounts of energy to its surroundings by radiation from the accretion disk and drives powerful gas outflows, which remove gas altogether from the galactic potential well (Cattaneo et al. 2009; Fabian 2012). The "radio mode," on the other hand, is prevalent mostly in low- to moderate-luminosity AGNs where the black hole accretes at a lower rate and the radio AGN present in the center of the galaxy deposits energy into the surrounding gas via jets or winds, heating it and suppressing star formation (Binney \& Tabor 1995; Ciotti \& Ostriker 2001, 2007; Bower et al. 2006; Croton et al. 2006; McNamara \& Nulsen 2007; Cattaneo et al. 2009; Ciotti et al. 2010; Fabian 2012; Heckman \& Best 2014; Yuan \& Narayan 2014). Direct observational evidence for this low-luminosity radio-mode, or "maintenance-mode," feedback is limited to several nearby clusters (Fabian 1994, 2012; Dunn \& Fabian 2006; Fabian et al. 2006; McNamara \& Nulsen 2007; Cattaneo et al. 2009). Evidence for this mechanism in more typical galaxies remains elusive.

Recently, Cheung et al. (2016) discovered a new class of quiescent galaxies, referred to as "red geysers," that show distinctive emission-line patterns showing gas outflows from the center and kinematic properties (explained in detail in Section 3.1.1) that may signal AGN maintenance-mode feedback in action. Based on spatially resolved information from the Sloan Digital Sky Survey IV (SDSS-IV) Mapping Nearby Galaxies at Apache Point Observatory (MaNGA) survey (Bundy et al. 2015), this class of quiescent galaxies appears to host large-scale winds of ionized gas that align with bisymmetric enhancements in the spatial distribution of strong emission lines like $\mathrm{H} \alpha$. Ionized emission extends throughout the entire galaxy with line ratios similar to low ionization emission region (LIER)-like galaxies (Belfiore et al. 2016). In addition to their enhanced bisymmetric line emission, the red geysers also exhibit gas kinematics consistent with outflowing winds. The gradient of the gas velocity field aligns with the position angle (PA) of the emission pattern but is largely misaligned with the major or minor axes derived from the stellar velocity field. The gas velocity values can reach $\sim 300 \mathrm{~km} \mathrm{~s}^{-1}$, a value that is difficult to explain by orbital motion from the galaxy's gravitational potential, considering the mass range of the galaxies.

Early-type galaxies with accreted disks, as studied by Lagos et al. (2015) and Chen et al. (2016), can show kinematic features similar to those of red geysers, but those features are formed due to a completely different phenomena. The accreted gas coming in from random directions will gradually align itself with either the major or minor axis through gravitational torques generated by the galaxy's potential well. Hence, while a misalignment in the velocity gradient of stars and gas can occur for these galaxies too, often the misalignment angle is $90^{\circ}$ or $0^{\circ} / 180^{\circ}$, depending on whether a polar disk or corotating/counterrotating disk is formed. Some galaxies with accreted disks might show $\mathrm{H} \alpha$ equivalent width (EW) distributions similar to those of red geysers. Cheung et al. (2016) rejected the disk interpretation through detailed Jeans anisotropic modeling (JAM; Cappellari 2008) of the prototypical red geyser with $99 \%$ confidence, which demonstrated that the gas velocity in this source is too high (the difference between the observed gas velocity and expected velocity from modeling being $\sim 100 \mathrm{~km} \mathrm{~s}^{-1}$ ) to be described by the orbital motion. Given similar high velocities and other common features shared among all the red geysers, outflowing winds emerge as a compelling interpretation (K. Bundy et al. 2018, in preparation), making the question of whether AGNs are capable of driving these winds particularly important.

A critical first step is to test the hypothesis that the red geyser population is more likely to host an active AGN compared to quiescent galaxies with similar global galaxy properties. For the prototypical red geyser named "Akira," Cheung et al. (2016) showed that the host galaxy has a weakly and/or radiatively inefficient supermassive black hole with a very low Eddington ratio $\left(\lambda=3.9 \times 10^{-4}\right)$, accreting mass from a low-mass companion galaxy. It was detected as a central radio point source.

The goal of this work is to search for radio-mode AGNs in the entire red geyser sample. We analyze stacked radio flux from the Very Large Array (VLA) Faint Images of the Radio Sky at Twenty-Centimeters (FIRST) survey and find a higher value of radio flux from the red geyser candidates than the comparison sample of quiescent galaxies. We have excluded possible star formation contamination and/or galaxies with embedded disks from our sample by using optical and infrared (IR) photometry. Section 2 describes the optical, IR, and radio data that we have used in this work. In Section 3.1, we discuss in detail the red geysers and the control sample chosen from the MaNGA local quiescent population. The technical details of radio-aperture photometry and the stacking analysis have been narrated in Section 3.2. The results thus obtained from the stacked radio flux are described in Section 4. The implications of the results are given in Section 5.

Throughout this paper, we assume a flat cosmological model with $H_{0}=70 \mathrm{~km} \mathrm{~s}^{-1} \mathrm{Mpc}^{-1}, \Omega_{m}=0.30$, and $\Omega_{\Lambda}=0.70$, and all magnitudes are given in the $\mathrm{AB}$ magnitude system.

\section{Data \\ 2.1. The MaNGA Survey}

Our sample comes from the ongoing SDSS-IV MaNGA survey (Bundy et al. 2015; Drory et al. 2015; Law et al. 2015; SDSS Collaboration et al. 2017; Yan et al. 2016; Blanton et al. 2017). MaNGA is an integral field spectroscopic survey that provides spatially resolved spectroscopy for nearby galaxies $(z \sim 0.03)$ with an effective spatial resolution of $2 . .5$ (FWHM). The MaNGA survey uses the SDSS $2.5 \mathrm{~m}$ telescope in spectroscopic mode (Gunn et al. 2006) and the two dualchannel BOSS spectrographs (Smee et al. 2013) that provide continuous-wavelength coverage from the near-UV to the nearIR: $3600-10000 \AA$. The spectral resolution varies from $R \sim 1400$ at $4000 \AA$ to $R \sim 2600$ at $9000 \AA$. An $r$-band 
signal-to-noise ratio $(\mathrm{S} / \mathrm{N})$ of $4-8 \AA^{-1}$ is achieved in the outskirts (i.e., $1-2 R_{e}$ ) of the target galaxies with an integration time of approximately $3 \mathrm{hr}$. MaNGA will observe roughly 10,000 galaxies with $\log \left(M_{*} / M_{\odot}\right) \gtrsim 9$ across $\sim 2700 \mathrm{deg}^{2}$ over its $6 \mathrm{yr}$ duration. In order to balance radial coverage versus spatial resolution, MaNGA observes two-thirds of its galaxy sample to $\sim 1.5 R_{e}$ and one-third to $2.5 R_{e}$. The MaNGA target selection is described in detail in Wake et al. (2017).

The raw data are processed with the MaNGA data reduction pipeline (DRP; Law et al. 2016). An individual row-by-row algorithm is used to extract the fiber flux and derive inverse variance spectra from each exposure, which are then wavelength calibrated, flat fielded, and sky subtracted. We use the MaNGA sample and data products drawn from the MaNGA Product Launch-5 (MPL-5) and Data Release 13. The data products are identical to those released as part of the SDSS Data Release 14 (DR14; Abolfathi et al. 2018). We use spectral measurements and other analyses carried out by a preliminary version of the MaNGA data analysis pipeline (DAP), specifically version $2.0 .2 .^{22}$ In brief, the data we use here are based on DAP analysis of each spaxel in the MaNGA data cubes. The DAP first fits the stellar continuum of each spaxel to determine the stellar kinematics using the penalized pixelfitting algorithm pPXF (Cappellari \& Emsellem 2004; Cappellari 2017) and templates based on the MILES stellar library (Falcón-Barroso et al. 2011). The templates are a hierarchically clustered distillation of the full MILES stellar library into 49 templates. This small set of templates provides statistically equivalent fits to those that use the full library of 985 spectra in the MILES stellar library. The emission-line regions are masked during this fit. The DAP then subtracts the result of the stellar continuum modeling to provide a (nearly) continuum-free spectrum that is used to fit the nebular emission lines. This version of the DAP treats each line independently, fitting each for its flux, Doppler shift, and width, assuming a Gaussian profile shape. This is different from the approach used by the DAP for DR15, which is to tie the velocities of all lines to a single value and to impose fixed flux ratios for the [O I], [O III], and [N II] line doublets. A detailed comparison of the results from the DR15 and MPL-5 versions of the DAP show that the different approach taken by the latter and used for our analysis has a negligible influence on our results. The final output from the DAP are gas and stellar kinematics, emissionline properties, and stellar absorption indices.

We use ancillary data drawn from the NASA-Sloan Atlas ${ }^{23}$ (NSA) catalog, which reanalyzes images and derives morphological parameters for local galaxies observed in SDSS imaging. It compiles spectroscopic redshifts, UV photometry (from GALEX; Martin et al. 2005), stellar masses, and structural parameters. We have specifically used spectroscopic redshifts and stellar masses from the NSA catalog.

\subsection{The FIRST Survey}

The radio data studied in this paper come from the VLA FIRST (Becker et al. 1995) survey, which obtained data at

\footnotetext{
${ }^{22}$ This version of the code will be made public in the upcoming SDSS Data Release 15 (DR15; Aguado et al. 2019, submitted). An overview of the DAP used for the DR15 and its products is described by K. Westfall et al. (2018, in preparation), and assessments of its emission-line fitting approach are described by F. Belfiore et al. (2018, in preparation).

23 http://www.nsatlas.org
}

frequency channels centered at 1.36 and $1.4 \mathrm{GHz}$ over $10,000 \mathrm{deg}^{2}$ in the north and south Galactic caps. The source detection threshold is $\sim 1 \mathrm{mJy}$, corresponding to a source density of $\sim 90$ sources $\mathrm{deg}^{-2}$. The FIRST images have 1 !. 8 pixels with a resolution of $\sim 5^{\prime \prime}$ and typical $\mathrm{rms}$ of $0.15 \mathrm{mJy}$. The astrometric accuracy of each source is $0 .{ }^{\prime \prime} 5-1^{\prime \prime}$ at the source detection threshold. Since the FIRST survey area was designed to overlap with the SDSS (York et al. 2000; Abazajian et al. 2009), most MaNGA targets have FIRST data coverage. For our sample of interest, $93 \%$ of the red geysers have FIRST coverage. However, the $1 \mathrm{mJy}$ threshold results in nondetections for most MaNGA galaxies.

For each pointing center, there are 12 adjacent single-field pointings that are coadded to produce the final FIRST image. Sources are extracted from the coadded reduced images and fit by two-dimensional Gaussians to derive peak flux, integrated flux densities, and size information. The current FIRST catalog is accessible from the FIRST search page. ${ }^{24}$

\section{3. $S D S S+\mathrm{WISE} S F R S$}

In order to assess possible contamination from obscured star formation, we have used the Chang et al. (2015) catalog to obtain IR-based star formation rates (SFRs). The catalog contains 858,365 galaxies within the SDSS spectroscopic sample as compiled in the New York University Value-added Galaxy Catalog (NYU-VAGC; Blanton et al. 2005; AdelmanMcCarthy et al. 2008; Padmanabhan et al. 2008) and crossmatched with the AllWISE source catalog from the Wide-field Infrared Survey Explorer (WISE). Unlike optical emission-line SFR estimates, Chang et al. (2015) utilized mid-IR data from full WISE photometry and employed a spectral energy distribution (SED) fitting technique to estimate stellar mass and SFR. Their modeling is based on the MAGPHYS library ${ }^{25}$ (MAGPHYS contains 50,000 stellar population template spectra and 50,000 $\mathrm{PAH}+$ dust emission template spectra) and is applied to all $z<0.2$ galaxies with good WISE photometry (FLAG_W $=1$ or 2 ) and good-quality SED fits (FLAG_CHI2 = 1). None of the objects in our sample feature AGN-like WISE colors; hence, it is unlikely that AGN contamination may be significantly biasing the SED-based SFR estimates. We have used the public Chang et al. (2015) catalogs. ${ }^{26}$ Details are given in Chang et al. (2015).

\section{Method}

The identification of red geysers is based on the optical resolved spectroscopic data from MaNGA. Section 3.1.1 describes the conditions and criteria that have been used to select our sample. Matched control sample galaxies have been selected from the full galaxy sample via the method discussed in Section 3.1.2. A third category of galaxies, which we call the "H $\alpha$-disturbed" class, as described in detail in Section 3.1.3, consists of galaxies that are not classified as geysers due to the absence of the characteristic bisymmetric pattern in the resolved optical emission map, but they show kinematic and emission-line properties that are quite different from those of typical quiescent galaxies. They have distinct regions of enhancement in the $\mathrm{H} \alpha-\mathrm{EW}$ map, along with highly spatially

\footnotetext{
${ }^{24}$ http://sundog.stsci.edu/cgi-bin/searchfirst. The full images are available at ftp://archive.stsci.edu/pub/vla_first/data.

25 http://www.iap.fr/magphys/

26 http://irfu.cea.fr/Pisp/yu-yen.chang/sw.html
} 
resolved gas velocity values in comparison to stellar velocity. The $\mathrm{H} \alpha$-disturbed class forms a separate category, distinct from both the red geyser and control samples. We perform aperture photometry (described in detail in Section 3.2) on the FIRST radio cutouts for all galaxies using an aperture size of $10^{\prime \prime}$ diameter to obtain the radio flux values and associated photometric errors. The galaxies that satisfy the condition $\mathrm{S} / \mathrm{N}>3$ are classified as radio-detected with a confidence level of $3 \sigma$. Since the detection threshold of the VLA FIRST survey is shallow ( $\sim 1 \mathrm{mJy}$ ), many galaxies might lie below the sensitivity limit. Section 3.2 describes the stacking analysis that allows us to constrain the average radio flux for samples of galaxies that are undetected individually. The median-stacked FIRST images for our sample provide a greater $\mathrm{S} / \mathrm{N}$ with a typical $\mathrm{rms}$ of $\approx 10 \mu \mathrm{Jy}$.

\subsection{Sample Selection}

In this section, we describe the identification of red geysers, selection of matched control sample galaxies, and characterization of the $\mathrm{H} \alpha$-disturbed galaxies.

\subsubsection{Red Geysers}

Red geysers are visually selected based on their characteristic features, as described in Cheung et al. (2016). Red geysers are red galaxies defined by rest-frame color NUV $-r>5$ (Figure 2). The specified UV-optical color cut selects predominantly quiescent galaxies (Salim et al. 2005, 2007, 2009). In MPL-5, 40\% of all targets fall in the quiescent category by the specific color cut. The SFR estimates derived from full SED fitting from optical to mid-IR data (Chang et al. $2015)$ are small for the red geysers (96\% of the sample has log SFR $\left[M_{\odot} \mathrm{yr}^{-1}\right]<-2$, shown later in Figure 8) to ensure quiescence and remove possible obscured star formation. Additionally, the spatially resolved map of the EW of the Dn4000 absorption feature is high, roughly $>1.4 \AA$, thus ensuring the absence of young stars in the galaxy. The red geysers show narrow bisymmetric patterns in the ionized gas emission as observed in the EW maps of strong emission lines like $\mathrm{H} \alpha$ and [O III]. These patterns line up approximately with the gaseous kinematic axis and show a misalignment with the stellar kinematic axis, but we pay close attention to cases where the misalignment of the stellar and gas velocity field is $0^{\circ}$, $180^{\circ}$, or $90^{\circ}$ in order to exclude embedded corotating, counterrotating, and polar gas disks. Another important defining property of the red geysers is that they have highly spatially resolved gas velocities that can reach a maximum value of $\sim 250-300 \mathrm{~km} \mathrm{~s}^{-1}$, much higher compared to stellar velocities, as well as high gas velocity dispersion values (maximum $\sim 200 \mathrm{~km} \mathrm{~s}^{-1}$ ). Hence, the observed second moment of the velocity $\left(V_{\mathrm{rms}} \equiv \sqrt{V^{2}+\sigma^{2}}\right)$ of the ionized gas typically exceeds the second-velocity moments of the stars by $80-100 \mathrm{~km} \mathrm{~s}^{-1}$, suggesting that the ionized gas kinematics in these galaxies cannot be explained by gravitationally bound orbits alone. For the prototype red geyser, Cheung et al. (2016) performed detailed JAM and used the dynamically constrained potential to get a rough estimate of the escape velocity, $V_{\text {esc }} \sim 400 \pm 50 \mathrm{~km} \mathrm{~s}^{-1}$. They found that roughly $15 \%-20 \%$ of the gas would exceed the escape velocity. A typical example of a red geyser is shown in Figure 1. Further details of the selection procedure will be described in K. Bundy at al. (in preparation).
Accreted gas disks in early-type galaxies (e.g., Chen et al. 2016) can sometimes produce similar gas velocity gradients like the red geysers due to rotation of the gaseous material in the disk. A few edge-on disks show a bisymmetric pattern in the EW map similar to the red geysers. Hence, we include a few steps in our visual identification to exclude galaxies with a visible disk component or dust lanes apparent in the optical SDSS image. We discard edge-on galaxies with axis ratio $b / a<0.3$. We also checked the galaxy specific stellar angular momentum $\left(\lambda_{\mathrm{Re}}\right)$ and ellipticity $(\epsilon)$ from the extensive catalog in Graham et al. (2018). Convincingly, we find that $95 \%$ of the red geysers are fast-rotator early-type galaxies. Our control sample galaxies are of a similar nature; $97 \%$ of them are fast rotators, according to Graham et al. (2018). Since the fast rotators have stellar disks and are axisymmetric, this implies that a gas disk cannot be in equilibrium if it is misaligned with the stellar kinematic PA, thus ruling out the possibility of disks being the source of the observed misalignment in the red geysers. Additionally, to avoid galaxies with rotating disks being included in the red geyser sample, any galaxy showing a very low value of average gas velocity dispersion through out the galaxy $\left(<60 \mathrm{~km} \mathrm{~s}^{-1}\right.$, which is roughly the average dispersion value observed in polar disks), has been discarded from our red geyser sample completely, even if it shows other convincing features of a red geyser.

As described in Cheung et al. (2016), the gas velocity fields of the red geysers are poorly fit by flexible disk rotation models. The error-weighted average residual, characterizing the goodness of fit, places the red geysers among the worst $5 \%$ of fitted MaNGA galaxies with disklike kinematics. The resolved spectral-line ratios land predominantly in the LINER region in the Baldwin, Phillips \& Telervich (BPT) diagram, predicting that the ionizing source is mostly post-asymptotic giant branch (AGB) stars and/or AGNs.

To summarize, the red geysers in our chosen sample have the following characteristic features.

1. Quiescent nature with rest-frame color NUV $-r>5$.

2. Bisymmetric emission feature in the $\mathrm{H} \alpha-\mathrm{EW}$ resolved map.

3. Rough alignment of the bisymmetric feature with the ionized gas kinematic axis but misalignment with the stellar kinematic axis.

4. High spatially resolved gas velocity values, typically reaching a maximum of $\pm 300 \mathrm{~km} \mathrm{~s}^{-1}$, which are greater than the stellar velocity values by at least a few factors.

5. Very low SFR, with a typical value of $\log$ SFR $\left[M_{\odot} \mathrm{yr}^{-1}\right]<-2$.

Currently, our sample has 84 red geysers, which account for $\approx 8 \%$ of quiescent MaNGA galaxies (defined as NUV $-r>5$; see Section 3.1.2).

\subsubsection{Control Sample}

We create a control sample of quiescent galaxies with NUV $-r>5$ (shown in Figure 2), which are matched in global properties but do not show the resolved geyser-like features described in Section 3.1.1.

For each red geyser, we match up to five unique quiescent galaxies with the following criteria:

1. $\left|\log M_{* \text {, red geyser }} / M_{* \text {, control }}\right|<0.2 \mathrm{dex}$,

2. $\left|z_{\text {red geyser }}-z_{\text {control }}\right|<0.01$, and 
RA: 119.12000

DEC: 50.28787

$z=0.13362$

MaNGA-ID: $1-378770$

Plate-IFU: 8715-3701

$\log M_{\text {., NSA }}: 11.46$

$n: 6.00$

NUV-r: 5.93

log SSFR: -12.39

$\log \sigma: 2.472$

b/a: 0.370

R_e (NSA): 8.88 arcs

R_e (GIM2D): 7.34 arcs

IFU size: 8.75 arcs
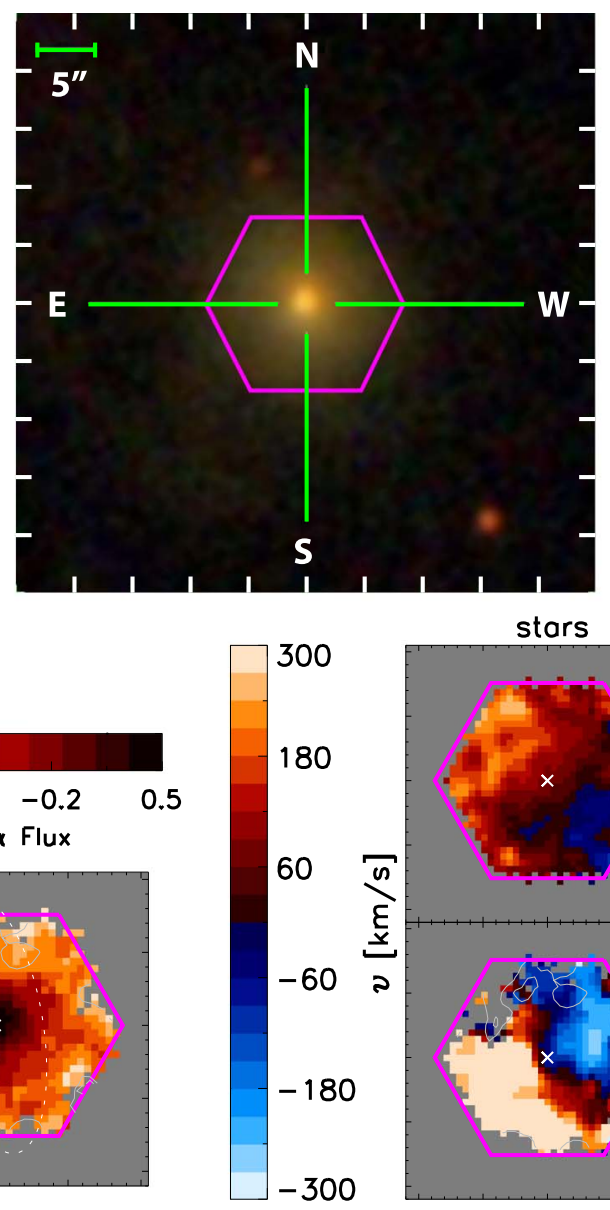
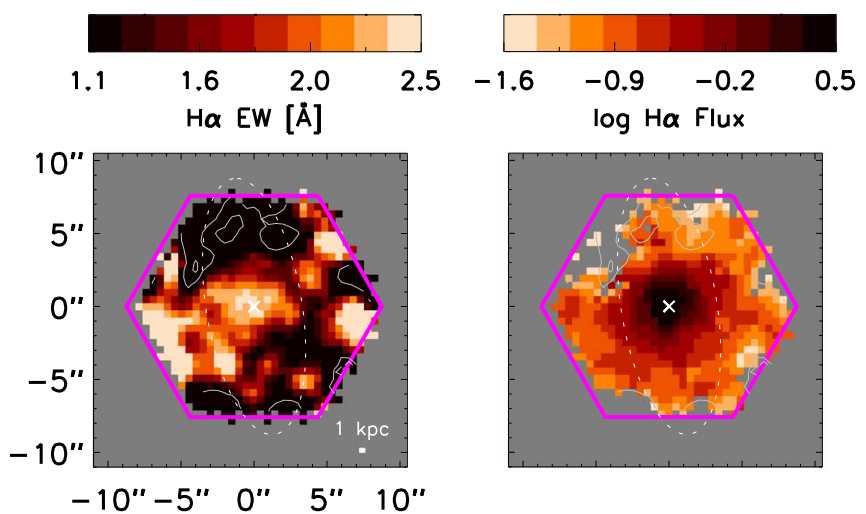

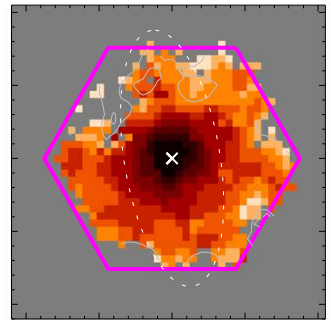

$\begin{array}{llll}-10 & -5 & 0 & \end{array}$ stars

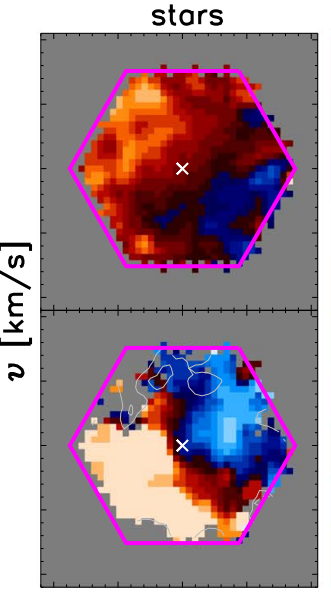

gas
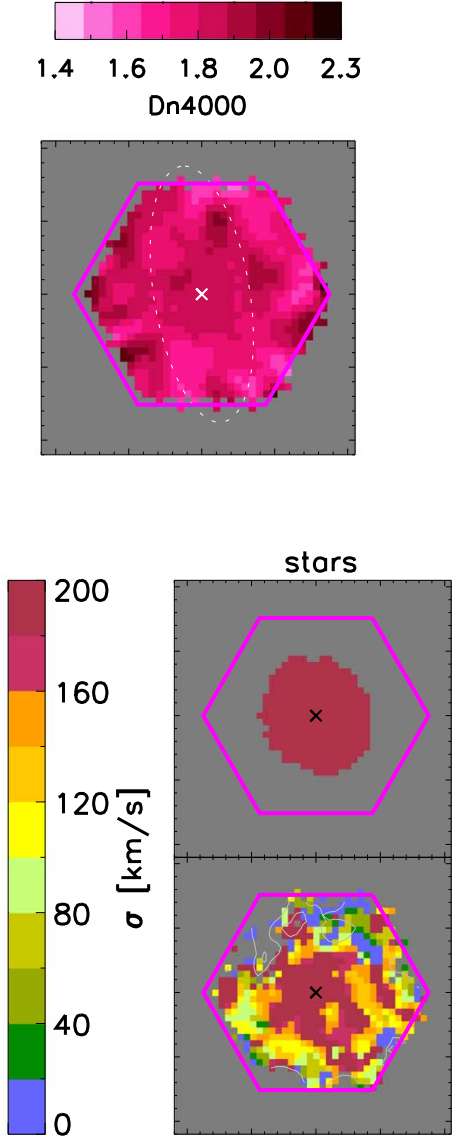

gas

Figure 1. Typical red geyser included in our sample. The data have been obtained from MaNGA integral field spectroscopic observations. The panel in the center shows the optical image of the galaxy (MaNGA-ID: 1-634825). The magenta hexagon marked in the image is the extent of the MaNGA fiber bundle. In the other panels, as labeled, we have shown the H $\alpha$ flux map, EW map, Dn4000 absorption map, and velocity maps of the gas and stars along with their dispersion. As described in Section 3.1.1, this galaxy satisfies all the conditions that we use to classify an object as a red geyser. Especially notable is the bisymmetric pattern in the EW map of $\mathrm{H} \alpha$, and the kinematic axis aligns perfectly with the gas velocity field.

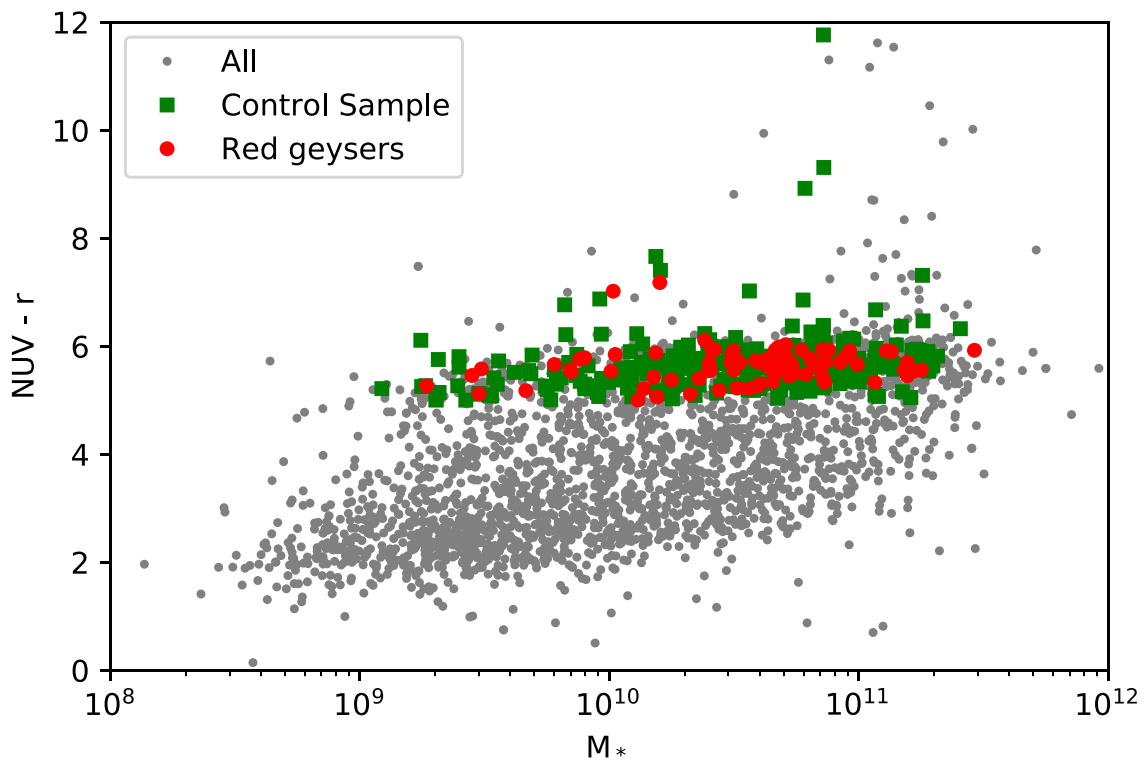

Figure 2. Rest-frame NUV $-r$ color vs. stellar mass $\left(\log M_{*}\right.$ ) diagram of the MaNGA sample, with the red geysers in red circles and the control galaxies in green squares. Quiescent galaxies are clustered in the upper part of the NUV $-r$ distribution; we define NUV $-r>5$ as a conservative boundary of quiescent galaxies. Galaxies with NUV $-r>8$ are undetected in the NUV data. 
RA: 258.02762

DEC: 57.50401

$z=0.02817$

MaNGA-ID: $1-24099$

Plate-IFU: $7991-1902$

$\log M_{\text {., NSA }}: 10.15$

$n: 3.89$

NUV-r: 5.50

log SSFR: -11.74

$\log \sigma: 2.110$

$\mathrm{b} / \mathrm{a}: 0.666$

R_e (NSA): 3.62 arcs

R_e (GIM2D): 3.76 arcs

IFU size: 6.25 arcs
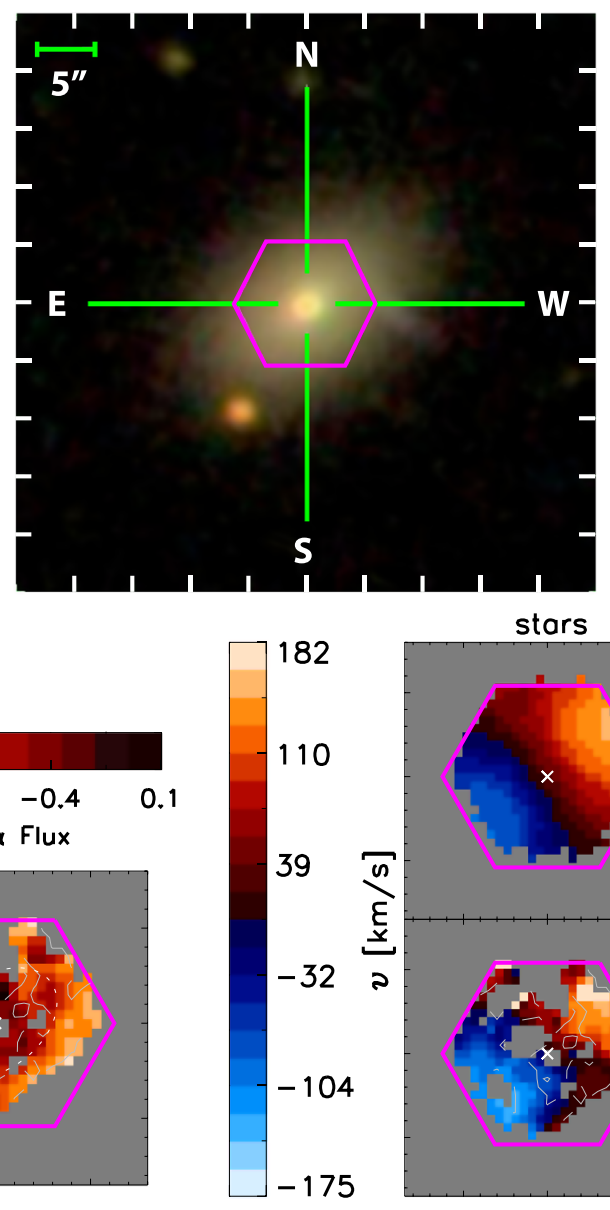

stars

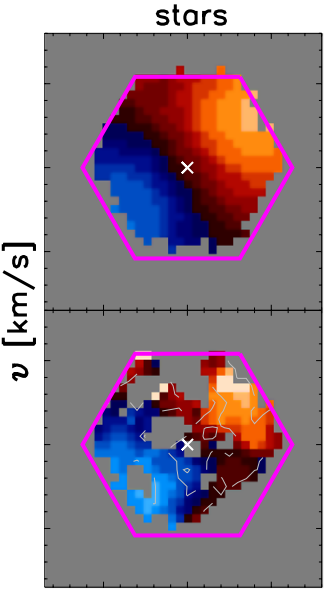

gas
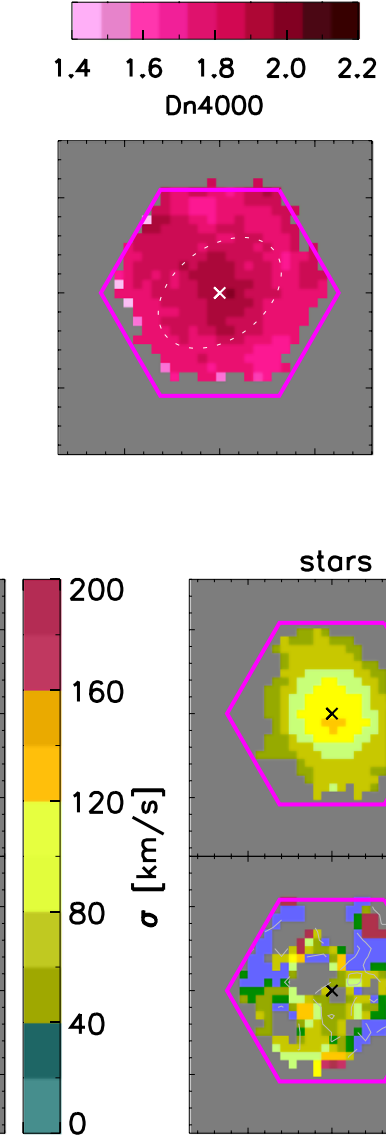

00

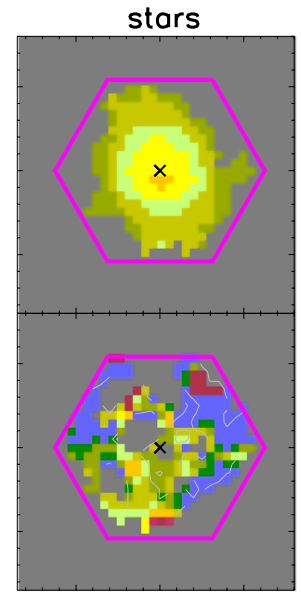

gas

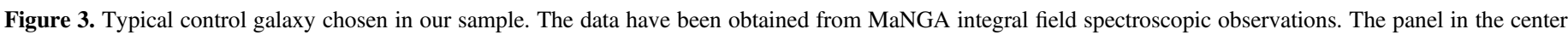

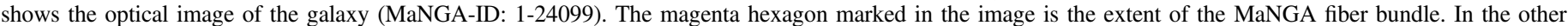

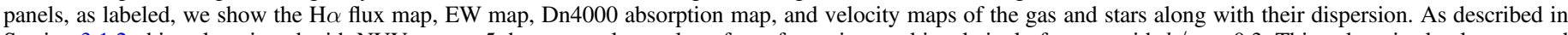

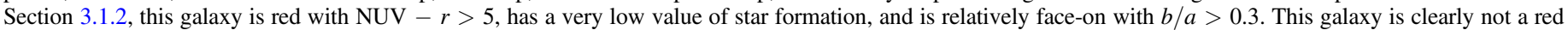
geyser, as it does not satisfy any of the red geyser features described in Section 3.1.1, so it can safely be included in the control sample.

\section{3. $\left|b / a_{\text {red geyser }}-b / a_{\text {control }}\right|<0.1$,}

where $M_{*}$ is the stellar mass, $z$ is the spectroscopic redshift, and $b / a$ is the axis ratio from the NSA catalog. Stellar mass and redshift have been shown to correlate with radio emission and thus must be controlled for (e.g., Condon 1984; Dunlop \& Peacock 1990; Best et al. 2005). We also control for axis ratio so that we do not compare potentially dust-reddened edge-on galaxies with the relatively face-on red geyser galaxies. This matching technique results in 260 unique control galaxies. Figure 3 shows an example of a typical quiescent galaxy from the control sample.

Figure 4 compares the global galaxy properties of the control sample and the red geysers. The red geysers (red) and control sample (blue) are well-matched in all four parameters-stellar mass, redshift, color, and axis ratio-as expected.

\subsubsection{Ho-disturbed Galaxies}

During the course of visual inspection, we discovered another category of galaxies that we will hereby refer to as "H $\alpha$-disturbed." Figure 5 shows an example. The gas content of these galaxies is comparable to that of the red geysers (median $\mathrm{H} \alpha$ EW value $>0.5 \AA$, similar to $\sim 0.8 \AA$ in the red geysers), but the $\mathrm{H} \alpha \mathrm{EW}$ maps do not show the clear bisymmetric patterns of a red geyser. They show twisted, disturbed $\mathrm{H} \alpha$ EW maps, sometimes with individual blobs of gas that are found throughout the galaxy. Of the total sample, $90 \%$ has spatially resolved gas velocity values reaching a maximum of $\sim 250 \mathrm{~km} \mathrm{~s}^{-1}$, which is high compared to the stellar velocities that lie within $\pm 60 \mathrm{~km} \mathrm{~s}^{-1}$. Some of them have high gas velocity dispersion, upward of $\sim 200 \mathrm{~km} \mathrm{~s}^{-1}$, as seen in the red geyser population. We found 60 such $\mathrm{H} \alpha-$ disturbed candidates from $\sim 900$ MaNGA quiescent populations, and we treat them as a separate third category different from both the red geyser and control samples.

\subsection{FIRST Radio Photometry and Stacking}

To obtain the radio flux, we perform aperture photometry on the FIRST cutouts for 78 out of 84 red geysers, 260 control galaxies, and 57 out of $60 \mathrm{H} \alpha$-disturbed galaxies that have FIRST coverage. We first determine which FIRST tile (of dimension $34.5 \times 46.5)$ a specific galaxy falls on. If a galaxy is located too close to the FIRST tile edge (less than $10^{\prime \prime}$ ), that galaxy is discarded. We extract a small cutout, $50 \times 50$ pixels wide (each pixel is 1."8), centered on the galaxy of interest. We use a circular aperture of $10^{\prime \prime}$ diameter centered on the galaxy and sum the radio flux values within. For our target galaxies, which are located roughly at redshift $\sim 0.03$, the $10^{\prime \prime}$ aperture 

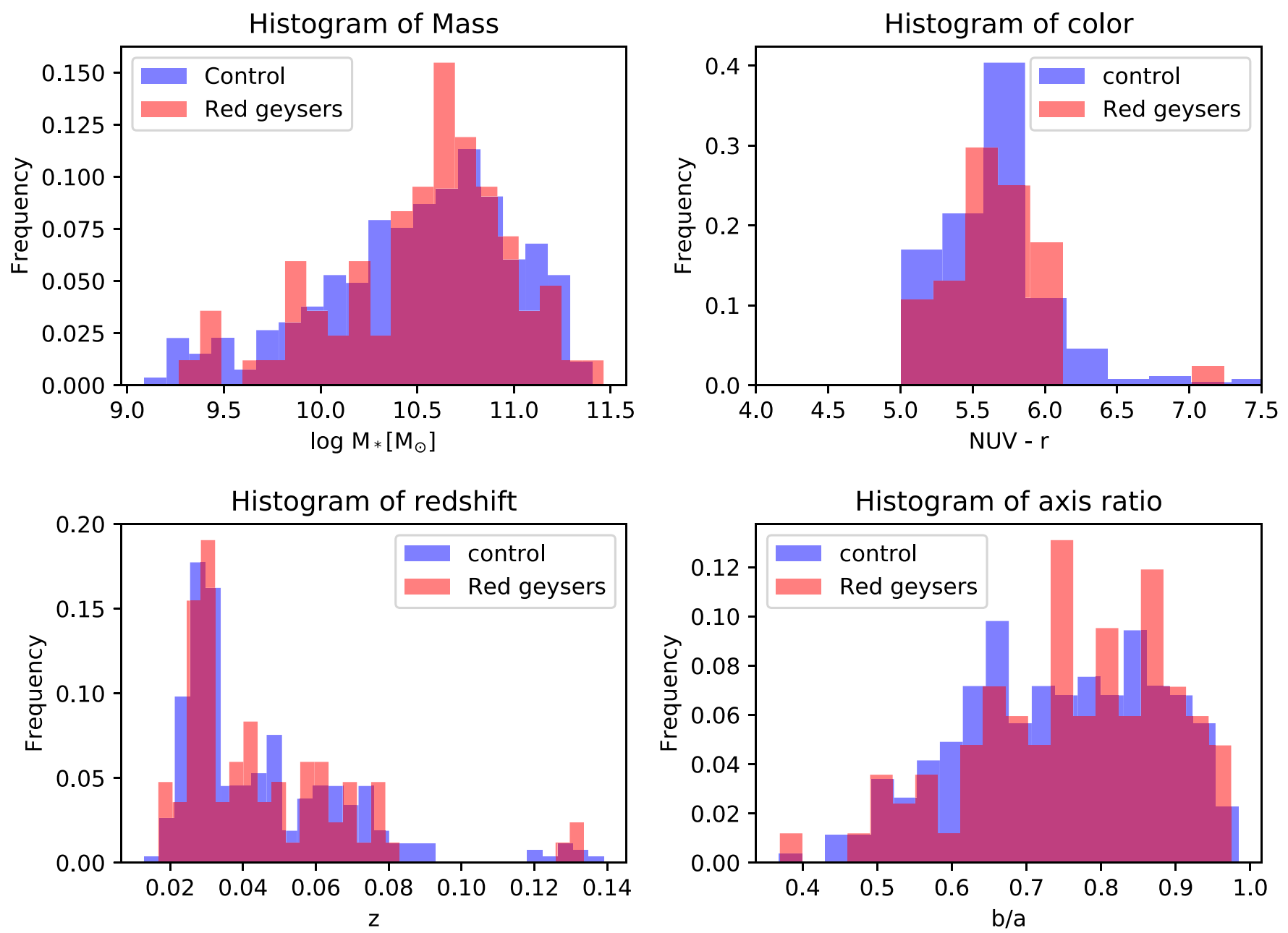

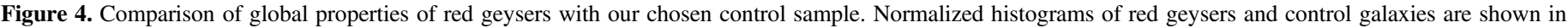

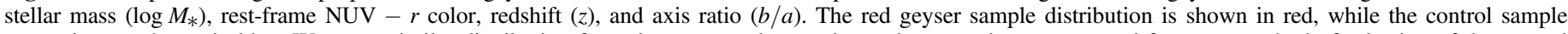

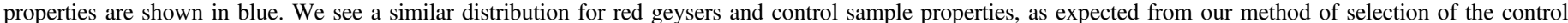
sample.

corresponds to $6 \mathrm{kpc}$ on the sky and hence is a reasonable choice for aperture size. We have defined the criteria for radio detection to be $\mathrm{S} / \mathrm{N}>3$. We then perform a median stack of the FIRST images associated with the three samples described in Section 3.1. To ensure that our results are not biased by a few radio-bright sources, we have made separate stacks of radio flux with the individually radio-detected sources removed.

We have also tested that our stacked radio signal is not an artifact of faulty FIRST tiles by median stacking random cutouts within a radius of $75^{\prime \prime}$ in the same FIRST tile where the galaxy is located. We would expect these "blank" stacks to have pure white noise with no radio signal.

Figure 6 shows the images of the median-stacked flux of these four samples: (1) the red geysers, (2) the control sample, (3) the non-radio-detected red geysers, and (4) the non-radiodetected control sample. The rightmost panel in both rows shows the blank stacks. Reassuringly, the blank stacks show no signal.

We perform additional separate stacks, controlling for ionized gas content and SFR in the control galaxies, to see their effect on the radio output. Details of our findings are given in Section 4.

In order to account for the photometric error, as well as the systematic error due to sample construction, we perform a bootstrap analysis on all of our samples. We construct 1000 random samples with replacement with the same size as each sample and compute the stacked radio flux as before. We take the standard deviation of the resulting flux distributions $(\sigma)$ to be the estimate of the error on the stacked flux measurements.

\section{Results}

\subsection{Radio Detection of Red Geysers versus Control Sample}

We have cross-matched FIRST radio detections with our sample of red geysers and control galaxies. Out of 78 red geysers, $12 \pm 3(\sim 15 \% \pm 4 \%)$ are found to be radio-detected, where the quoted errors are obtained from standard Poisson statistics. Among the control sample, $14 \pm 4$ out of 260 are radio-detected, with a detection fraction of $\sim 5 \% \pm 1.5 \%$. Red geysers show a three times higher radio detection rate compared to our control sample with a significance level of $5 \sigma$. We also find that the radio-detected red geysers make up an appreciable fraction $(\sim 10 \%)$ of the red MaNGA galaxies that are radio-detected by the FIRST survey. This fraction increases to $\sim 20 \%$ when the $\mathrm{H} \alpha$-disturbed category galaxies are included along with the red geyser population. If we limit our sample to $\log \left(M_{\star} / M_{\odot}\right)<11$, the detection rate of red geysers and $\mathrm{H} \alpha$-disturbed galaxies goes up to $40 \%$. 
RA: 116.78572

DEC: 40.39294

$z=0.07646$

MaNGA-ID: $1-43933$

Plate-IFU: 8140-3704

$\log M_{\text {., NSA: }} 10.81$

$n: 6.00$

NUV-r: 6.27

log SSFR: -11.90

$\log \sigma: 2.291$

b/a: 0.919

R_e (NSA): 4.76 arcs

R_e (GIM2D): 5.98 arcs

IFU size: 8.75 arcs
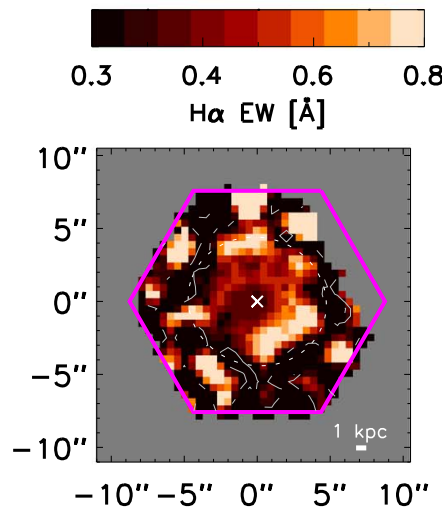

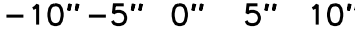

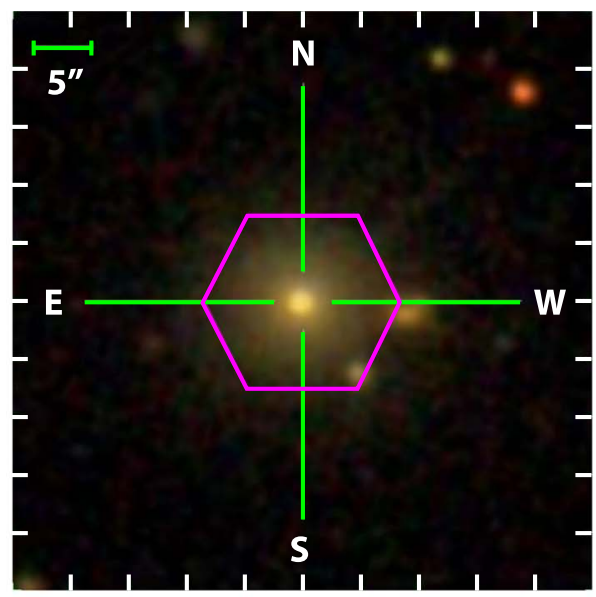

stors

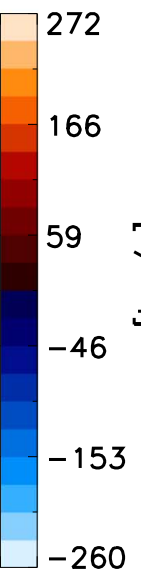

$-200$
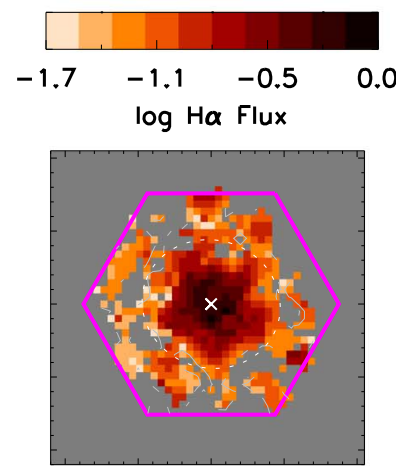

Figure 5. Typical disturbed galaxy as described in Section 3.1.3. The data have been obtained from MaNGA integral field spectroscopic observations. The panel in the center shows the optical image of the galaxy (MaNGA-ID: 1-43933). The magenta hexagon marked in the image is the extent of the MaNGA fiber bundle. In the other panels, as labeled, we have shown the H $\alpha$ flux map, EW map, Dn4000 absorption map, and velocity maps of the gas and stars along with their dispersion. As described in Section 3.1.3, this galaxy cannot be called a promising geyser candidate because of the lack of the signature bisymmetric pattern, but the kinematics indicate a difference from the ordinary control sample. It has been classified as a third " $\mathrm{H} \alpha$-disturbed" category to separate it from the geyser and the control sample population.

\subsection{Stacked Radio Activity of Red Geysers versus the Control Sample}

Figure 7 shows the first main result of our analysis. We compare the median-stacked radio fluxes of the red geysers (red circles) with those of the control sample (blue squares). Data points on the side marked "All" indicate the median fluxes when the entire sample of geysers and control samples is included in the stack. On the side labeled "Radio Nondetections," we have excluded radio-bright red geysers and control galaxies. We see that for both cases, the red geyser radio fluxes obtained from median stacking are three times higher than the control sample at greater than $99.99 \%$ confidence $(>5 \sigma)$.

We additionally control for the presence of ionized gas in our sample. We obtain $\mathrm{H} \alpha \mathrm{EW}$ measurements from the MaNGA DAP. The mean value obtained by averaging the EW $(\mathrm{H} \alpha)$ values of all spaxels in a particular galaxy within 1.5 effective radii is used as the mean EW value and a proxy for ionized gas content. The control galaxies show an average value of $0.3 \AA$, somewhat lower than the corresponding $0.8 \AA$ seen in the red geyser sample. To compare against galaxies with similar EW values, we select an additional control sample with EW $>0.5 \AA$ (yellow diamonds). We see that even the radio stack of control galaxies having a comparable level of ionized gas has a value about three times less than that of the red geyser stack. In addition to that, the stacked radio flux for the control galaxies with ionized gas does not show much difference for the "All" and "Radio Nondetections" samples, which implies that the presence of a higher amount of ionized gas in the control sample does not necessarily affect the radio detection rate.

The detailed implications of these findings are summarized in Section 5.

\subsection{Dusty Star Formation}

As described in Section 3.1.1, we set a color cut of rest-frame NUV $-r>5$ and exclude edge-on galaxies with $b / a<0.3$ to avoid possible radio contamination by star formation to the radio flux. However, UV wavelengths are susceptible to dust attenuation and may not reveal heavily obscured star formation (e.g., Calzetti 2001). Here we use the SDSS + WISE Chang et al. (2015) catalog for obtaining SFR based on IR fluxes that are sensitive to dusty star formation. Chang et al. (2015) utilized the full WISE photometry to model the SED in the optical through mid-IR bands and obtained updated measures of mass and SFR. 

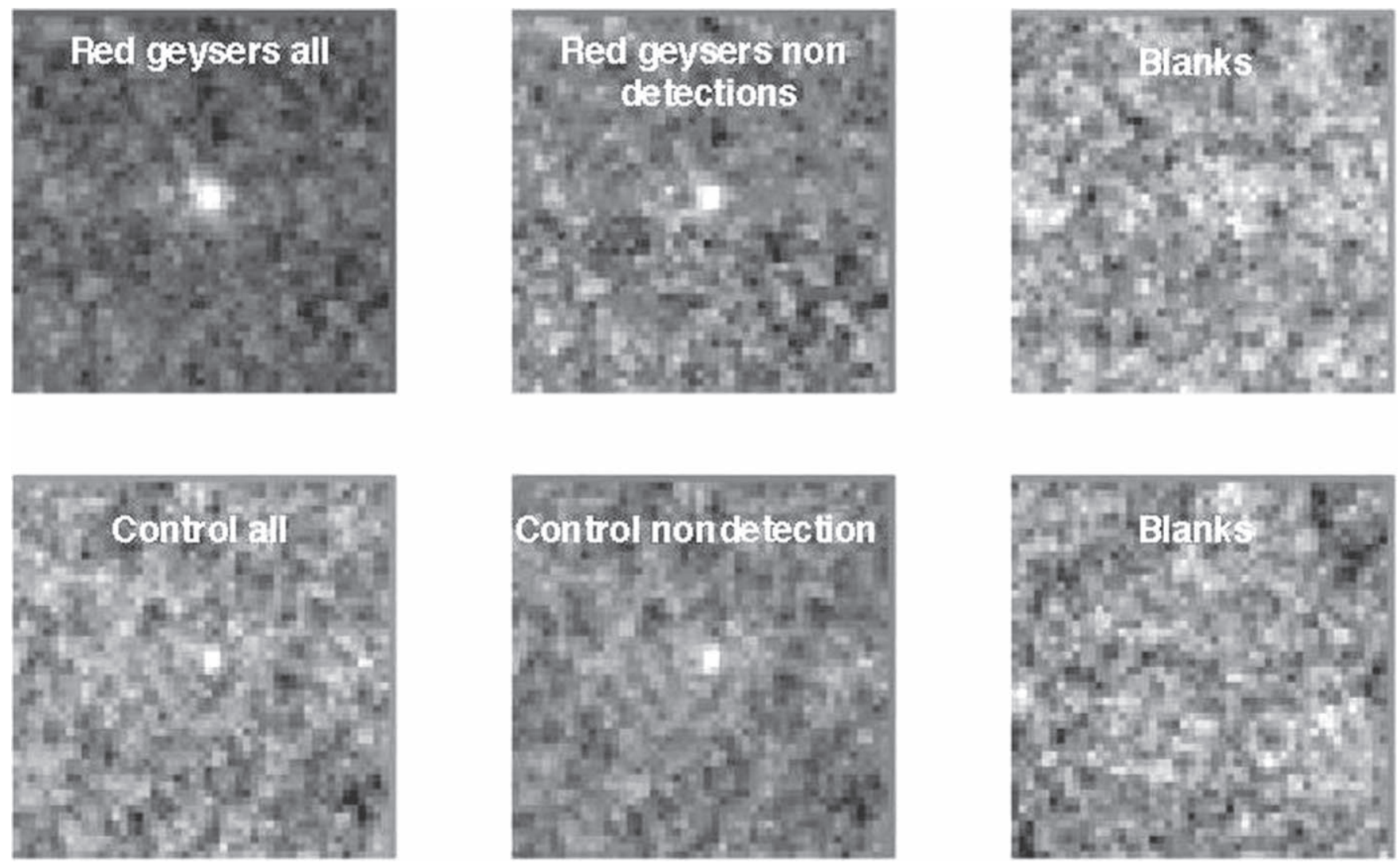

Figure 6. Median-stacked images of red geysers (top panels) and the control sample (bottom panels). The middle panels show the non-radio-detected stacked images for the red geysers (top) and the control (bottom), where all radio-detected sources have been excluded. The blank stacks are shown in the top right and bottom right panels.

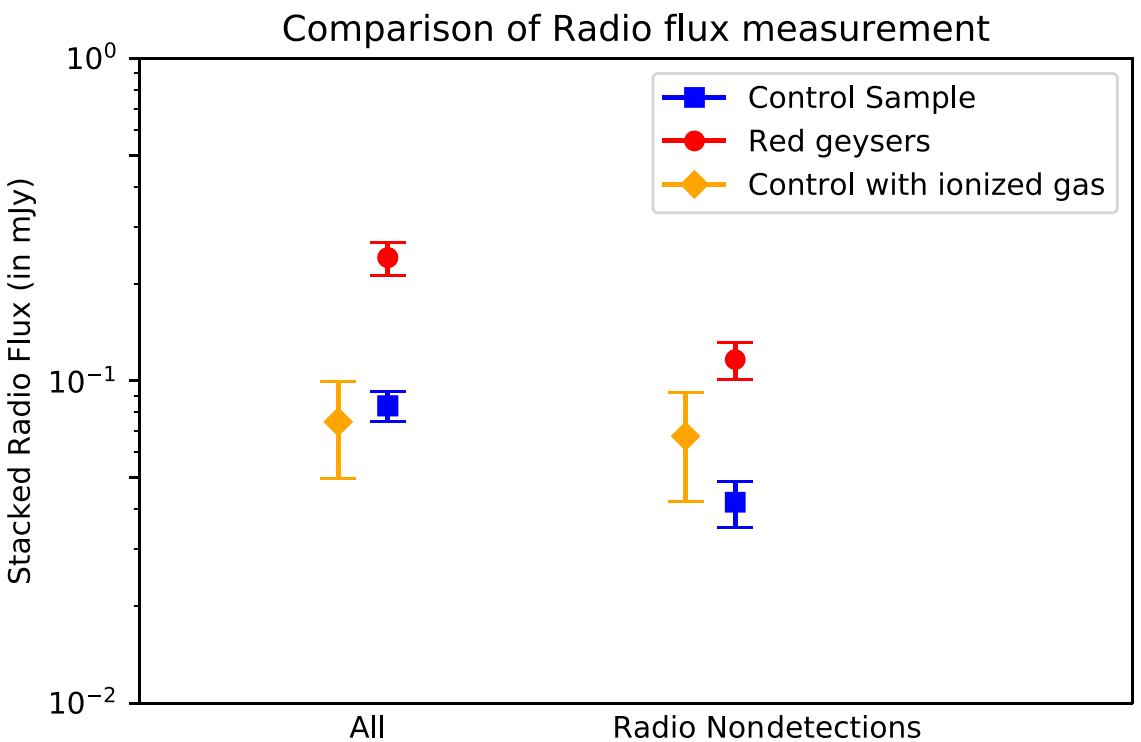

Figure 7. Median-stacked radio flux obtained from the stacked sample of red geysers (red circles) and the control sample (blue squares). "All" represents the stacks where the entire sample has been included for both red geysers and control, while "Radio Nondetections" indicates the stacks where the individually radio-detected sources have been removed. The condition of radio detection of a source has been defined as S/N > 3. "Control with ionized gas," marked with yellow diamonds, shows a specific subset of control galaxies when we additionally controlled for ionized gas (described in detail in Section 4). The red geyser sample shows an enhanced radio flux compared to the control sample galaxies, and the presence of a higher amount of ionized gas in the control sample does not necessarily affect the radio detection rate. The spaxel-by-spaxel EW information has been obtained from the MaNGA DAP and averaged over the spatial extent of 1.5 effective radii to obtain the mean EW value for a particular galaxy.

Figure 8 shows the $\log$ SFR versus $\log M_{\star}$ diagram of the galaxies from the Chang et al. (2015) catalog. We see that the majority of red geyser and control galaxies lie in the non-star-forming region, with low values of SFR. To ensure that our result is not affected by radio contamination from dusty star formation, we have redone the stacking analysis after 


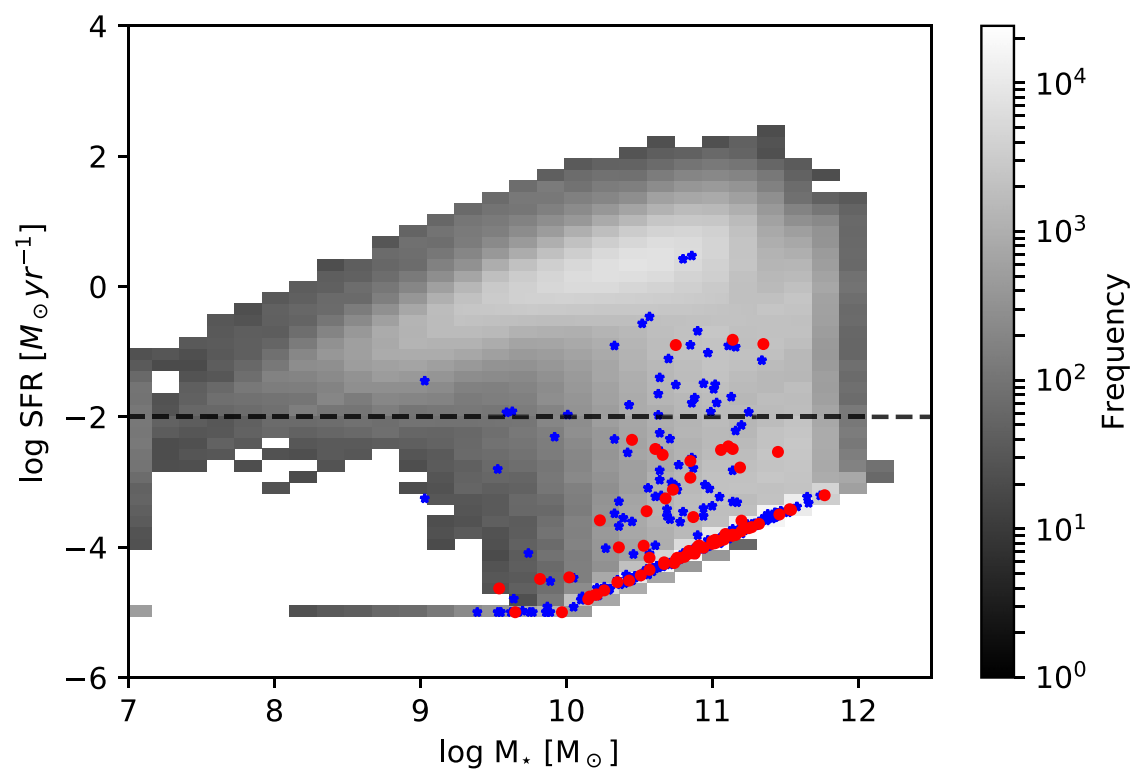

Figure 8. The $\log$ SFR vs. $\log M_{\star}$ as obtained from the SDSS + WISE catalog of Chang et al. (2015). The gray 2D histogram shows all the galaxies in the catalog with $0.01<z<0.1$. The red circles and blue stars signify red geyser and control sample galaxies, respectively. Most of the galaxies in our chosen sample have a low log SFR value, $<-2 M_{\odot} \mathrm{yr}^{-1}$.

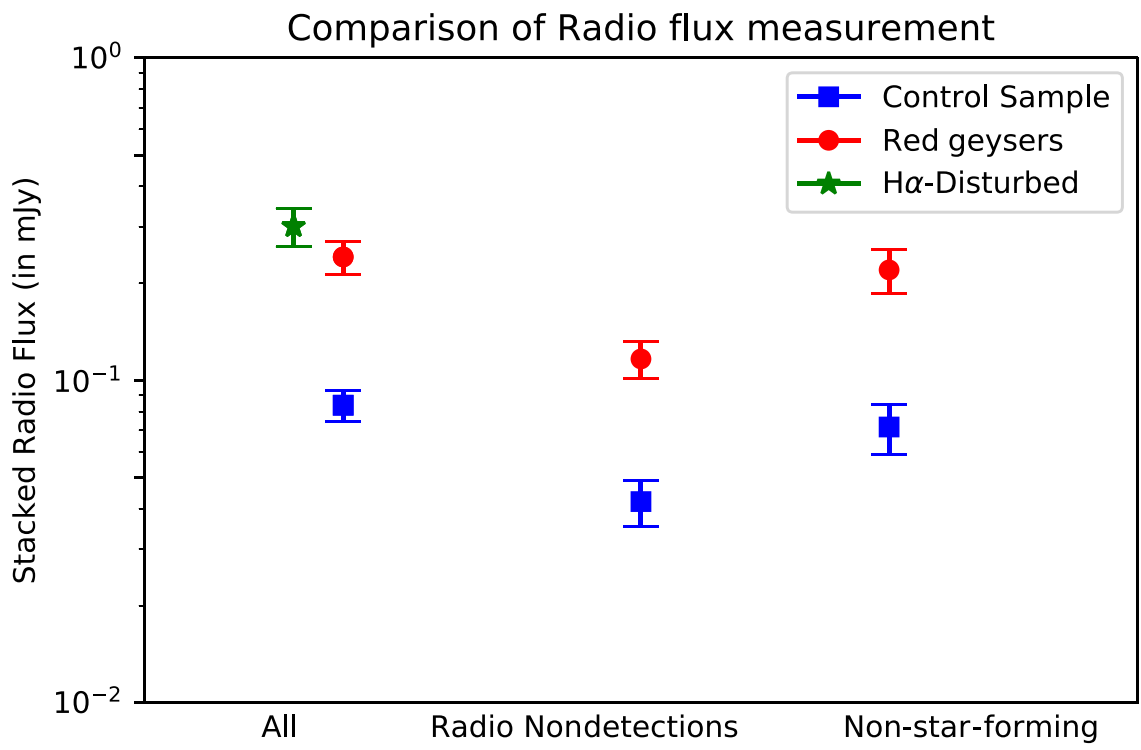

Figure 9. Median-stacked radio flux obtained from the stacked sample of red geysers (red), H $\alpha$-disturbed galaxies (green), and the control sample (blue). The leftmost region shows the stacks for the entire sample of red geysers (red circle), the control sample (blue square), and the H $\alpha$-disturbed category (green star). The "Radio Nondetections" region shows the stacked radio flux for the geysers and the control sample where the individually radio-bright ones, satisfying the criteria $\mathrm{S} / \mathrm{N}>3$, have been removed. The red geyser and the control sample have been cross-matched with the SDSS+WISE catalog of Chang et al. (2015). Galaxies with $\log$ SFR $>-2 M_{\odot} \mathrm{yr}^{-1}$ have been removed and restacked. They constitute the "Non-star-forming" category shown in the rightmost region of the plot. In all cases, the median-stacked radio flux is higher for the red geyser sample compared to the control sample by $>5 \sigma$.

excluding galaxies that have $\log \mathrm{SFR}\left[M_{\odot} \mathrm{yr}^{-1}\right]>-2$. This cut removes three red geysers and 30 control sample galaxies. Figure 9 shows the median-stacked radio flux in the region labeled "Non-star-forming." We conclude that our results are not affected by contamination from dusty star formation.

WISE colors can be used to detect strong nuclear heating associated with bright AGNs or quasars at the center of the host galaxy. According to Yan et al. (2013), $W 1(3.4 \mu \mathrm{m})-$ $W 2(4.6 \mu \mathrm{m})>0.8$ presents an efficient mid-IR color-based selection criterion for luminous AGNs and quasars. Most of the red geysers and control sample have $0.6<W 1-W 2<0.7$, with very few (one or two) having a value $>0.8$. This lends confidence to the ability of the WISE data to constrain obscured star formation in these galaxies, as there is no AGN contamination present in the SEDs of these galaxies. We trust the SED-based SFRs from Chang et al. (2015).

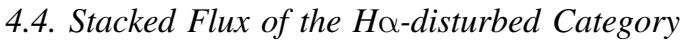

In Figure 9, the stacked flux for the galaxies in the disturbed category is shown with green stars. Remarkably, these galaxies show a slightly higher value of median-stacked radio flux than the red geysers. The disturbed EW maps and high gas velocity dispersions revealed by MaNGA data correlate with enhanced 
radio flux. We will discuss the implication of this finding in Section 5 .

\section{Discussions and Conclusion}

We have performed a radio-stacking analysis of 78 red geysers selected from the MaNGA survey that have FIRST coverage and compared their median radio flux with a sample of quiescent galaxies matched in global integrated properties but not classified as red geysers. The red geyser galaxies show significantly higher radio fluxes than the control galaxies, despite the fact that the red geyser selection is based on optical data alone. This suggests a physical link between the optical features that identify red geysers and the presence of enhanced AGN activity, lending further support to the argument that AGN-driven winds are responsible.

We have made several subdivisions based on different physical criteria to check our results.

1. We have performed the stacking for all galaxies in both the red geyser and control samples.

2. We have performed the stacking for samples in which the radio-detected sources are removed so that a few bright sources do not dominate the median-stacked radio flux value.

3. We have performed the stacking for galaxies with similar levels of ionized gas by imposing a cut on $\mathrm{EW}(\mathrm{H} \alpha)$ value.

4. We have performed the stacking for samples that exclude galaxies that show a high value of star formation from SDSS+WISE.

In all cases, red geysers exhibit elevated radio flux values.

Given our conservative NUV - $r$ color cut, the use of WISE mid-IR data (Section 4.3), and the absence of star-forming H II regions from resolved BPT diagrams, we can rule out star formation as the explanation for this enhanced radio flux. The other most likely sources are AGN activity or supernova remnants. SN Ia remnants can induce radio synchrotron emission from shock-accelerated cosmic rays. However, in our case, they are unlikely to be responsible for the increased radio signal in the red geyser sample because our selection criteria do not involve any factors that may enhance or suppress the SN Ia rate. We have controlled primarily for the $M_{*}$, restframe NUV $-r$ color, and age of the galaxy. Thus, there should be no difference in the frequency of SN Ia remnants between the red geysers and the control sample.

We conclude that the enhanced radio emission of red geysers is due to the presence of radio-mode AGNs. To confirm this statement, we compare the expected SFR from the average radio luminosity from the entire red geyser sample with the observed SFR derived from full SED fitting of optical mid-IR data from the Chang et al. (2015) catalog. The mean radio luminosity density $\left(L_{1.4 \mathrm{GHz}}\right)$ obtained from the stacked integrated flux density is $L_{1.4 \mathrm{GHz}} \sim 2 \times 10^{21} \mathrm{~W} \mathrm{~Hz}^{-1}$ (obtained by averaging the values of the radio luminosity of the red geysers in two mass bins, shown by black stars in Figure 10). From the best-fit relation between $1.4 \mathrm{GHz}$ radio continuum luminosity and the Balmer decrement-corrected $\mathrm{H} \alpha$ (Brown et al. 2017), we obtain a corresponding $\mathrm{H} \alpha$ luminosity of $\sim 1.3 \times 10^{41} \mathrm{erg} \mathrm{s}^{-1}$. Using the known relation between SFR and $\mathrm{H} \alpha$ luminosity (Kennicutt et al. 2009; Brown et al. 2017), assuming a Kroupa initial mass function (Kroupa \& Weidner 2003), we obtain an expected SFR from this radio emission of $\sim 1 M_{\odot} \mathrm{yr}^{-1}$. However, the observed mean SFR from the sample, as shown in Figure 8 , is $\sim 10^{-3} M_{\odot} \mathrm{yr}^{-1}$, which is much lower than expected, thus confirming AGNs as the primary source of radio emission, rather than star formation in the red geysers. The AGN feedback can induce radio emission through radio jets (Zensus 1997; Falcke \& Biermann 1999), advection-dominated accretion flows (Narayan et al. 1995, 2000), and/or winds (Jiang et al. 2010).

It stands to reason that the AGNs in the red geysers may act as the central powerhouse driving the ionized gas winds that signal the red geyser phenomenon.

It is interesting to consider how the $\mathrm{H} \alpha$-disturbed galaxies fit in this context. These galaxies show a comparable (within the uncertainty) or slightly higher value of stacked radio flux compared to the red geysers. All of them show significant gas blobs in the $\mathrm{H} \alpha$ EW maps. Some of them can be potential geyser candidates or relics from mergers or tidal interactions with other galaxies. The complex gas morphology might be a product of a multiphase and clumpy ISM ionized by the central AGN. These blobs may form out of the geyser wind material after the central engine shuts down. They may also result from a less stable accreting source. Given the uncollimated and chaotic distribution of ionized gas, it seems unlikely that the cool inflowing of material from a galactic encounter is responsible. There is also no indication that the $\mathrm{H} \alpha$-disturbed galaxies have recently undergone a merger or interaction. Clearly, more work is needed to understand them.

We would also like to highlight the handful of control galaxies with clear radio detections that are not classified as red geysers or $\mathrm{H} \alpha$-disturbed. These galaxies likely host a central active nucleus and exhibit significant emission-line flux. They may mean any of the following.

1. Our red geyser sample based on visual inspection is not a complete sample of AGN-driven ionized winds. Red geysers may be a special type of AGN wind phenomenon.

2. The AGNs in the control sample are too weak to drive out sufficient gas for detection at large radii.

3. A time lag may exist between AGN triggering and the development of a large-scale wind. Those AGN-hosted control galaxies may not be in the red geyser phase at the current epoch but may have passed through this phase in the past or might in the future.

Figure 10 shows the variation of radio luminosity $\left(L_{1.4 \mathrm{GHz}}\right)$ with stellar mass $\left(M_{\star}\right)$ for all FIRST radio-detected quiescent galaxies in the MaNGA sample. We see that the radio AGN population occupies two distinct regions in the plot, depending on the properties of the host quiescent galaxies. Radio AGNs in the galaxies showing optical emission-line features (red geysers and $\mathrm{H} \alpha$-disturbed galaxies) are found mostly at $\log \left(M_{\star} / M_{\odot}\right)<11$ (the low-mass end of the typical quiescent galaxy population), while the radio detection rate overall is higher for $\log \left(M_{\star} / M_{\odot}\right)>11$ by almost a factor of 1.4 compared to the lower-mass galaxies. One possibility is that red geysers and "radio galaxies" represent different AGN populations with different associated accretion histories and fueling mechanisms. Alternatively, the declining presence of wide-scale ionized gas at a higher stellar mass (Belfiore et al. 2017) may simply hide the existence of AGN-driven winds at higher masses. 


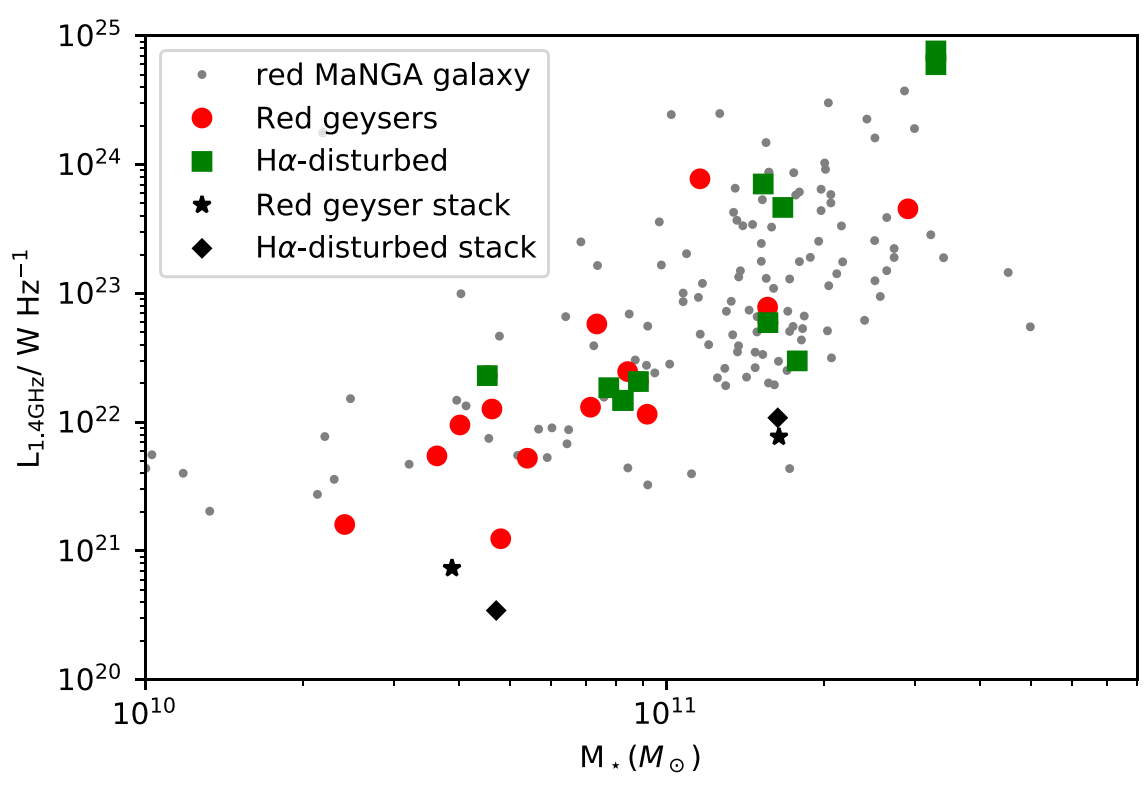

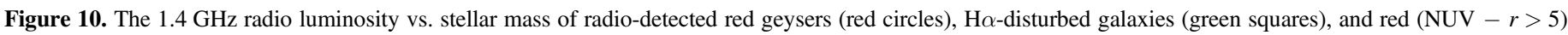

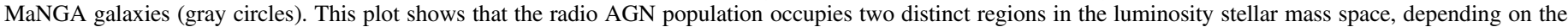

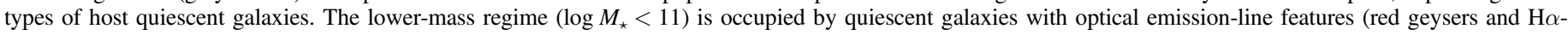

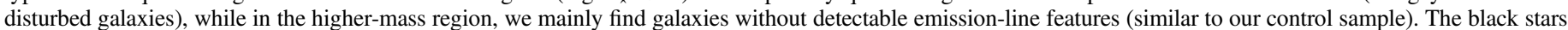

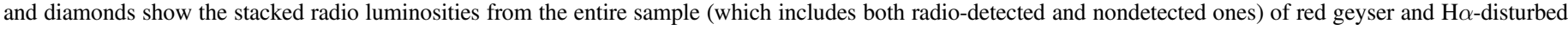
galaxies, respectively, in two mass bins.

We can gain further insight by considering the average luminosities from our stacked samples in two stellar mass bins. Using the median redshift in each mass bin, we overplot the average luminosity of red geysers and $\mathrm{H} \alpha$-disturbed galaxies in Figure 10 (shown with black stars and diamonds, respectively). The average luminosity has been obtained from the stacked radio flux that includes both radio-detected and nondetected sources. While radio-detected sources show a strong mass dependence, the average radio luminosity associated with red geysers shows a slightly weaker dependence with stellar mass. This suggests that a different kind of accretion physics may be at play.

Considering the two red geyser mass bins in Figure 10, we see that the typical radio power of the red geysers is $\sim 10^{21} \mathrm{~W} \mathrm{~Hz}^{-1}$ (shown in Figure 10 by black stars). From the best-fit linear relation between jet mechanical energy and radio power from Heckman \& Best (2014), we get an estimate of the jet kinetic energy to be $3 \times 10^{41} \mathrm{erg} \mathrm{s}^{-1}$. The AGNs in the red geysers are low-luminosity sources, and their mechanical energy will be confined predominantly to the size scales of the host galaxy halo. Also, according to our interpretation, the short-lived geyser phase possibly occurs in any red-sequence galaxy; hence, the "duty cycle" represents the number of galaxies with an active red geyser phase in the present. Hence, if we assume that the observed fraction of red geysers represents their duty cycle, then these phenomena are present $10 \%$ of the time (higher, if we consider $\left.\log \left(M_{\star} / M_{\odot}\right)<11\right)$. Multiplying this duty cycle by the typical jet kinetic power yields $\sim 3 \times 10^{40} \mathrm{erg} \mathrm{s}^{-1}$, an estimate of the AGN power averaged over long timescales. We can compare this to the cooling rate implied from the X-ray gas in this stellar mass range (O'Sullivan et al. 2001; Best et al. 2006), which is similar, $\sim 10^{40} \mathrm{erg} \mathrm{s}^{-1}$. This similarity provides further evidence that red geysers may play an energetically interesting role in the suppression of gas cooling and star formation at late times.
NR thanks Professor Puragra Guhathakurta for helpful comments and discussions. RAR acknowledges CNPq and FAPERGS.

Funding for the Sloan Digital Sky Survey IV has been provided by the Alfred P. Sloan Foundation, the U.S. Department of Energy Office of Science, and the Participating Institutions. The SDSS-IV acknowledges support and resources from the Center for High-Performance Computing at the University of Utah. The SDSS website is www.sdss.org.

The SDSS-IV is managed by the Astrophysical Research Consortium for the Participating Institutions of the SDSS Collaboration, including the Brazilian Participation Group, the Carnegie Institution for Science, Carnegie Mellon University, the Chilean Participation Group, the French Participation Group, Harvard-Smithsonian Center for Astrophysics, Instituto de Astrofísica de Canarias, The Johns Hopkins University, Kavli Institute for the Physics and Mathematics of the Universe (IPMU)/University of Tokyo, the Korean Participation Group, Lawrence Berkeley National Laboratory, Leibniz Institut für Astrophysik Potsdam (AIP), Max-Planck-Institut für Astronomie (MPIA Heidelberg), Max-Planck-Institut für Astrophysik (MPA Garching), Max-Planck-Institut für Extraterrestrische Physik (MPE), the National Astronomical Observatories of China, New Mexico State University, New York University, the University of Notre Dame, Observatário Nacional/MCTI, The Ohio State University, Pennsylvania State University, Shanghai Astronomical Observatory, the United Kingdom Participation Group, Universidad Nacional Autónoma de México, the University of Arizona, the University of Colorado Boulder, the University of Oxford, the University of Portsmouth, the University of Utah, the University of Virginia, the University of Washington, the University of Wisconsin, Vanderbilt University, and Yale University. 


\section{ORCID iDs}

Namrata Roy (1) https://orcid.org/0000-0002-4430-8846 Kevin Bundy (i) https://orcid.org/0000-0001-9742-3138 Edmond Cheung (1) https://orcid.org/0000-0001-8546-1428 Wiphu Rujopakarn iㅣ https://orcid.org/0000-0002-0303-499X Michele Cappellari (i) https://orcid.org/0000-0002-1283-8420 Renbin Yan 니 https://orcid.org/0000-0003-1025-1711 Tim Heckman (1) https://orcid.org/0000-0001-6670-6370 Matthew Bershady (i) https://orcid.org/0000-0002-3131-4374 Niv Drory (i) https://orcid.org/0000-0002-7339-3170 Kate Rubin (i) https://orcid.org/0000-0001-6248-1864 David Law (1) https://orcid.org/0000-0002-9402-186X Kai Zhang ๑ https://orcid.org/0000-0002-9808-3646 Joseph Gelfand (1) https://orcid.org/0000-0003-4679-1058 Dmitry Bizyaev (1) https://orcid.org/0000-0002-3601-133X David Wake iㅏ https://orcid.org/0000-0002-6047-1010 Karen Masters $\odot$ https://orcid.org/0000-0003-0846-9578

\section{References}

Abazajian, K. N., Adelman-McCarthy, J. K., Agüeros, M. A., et al. 2009, ApJS, 182, 543

Abolfathi, B., Aguado, D. S., Aguilar, G., et al. 2018, ApJS, 235, 42 Acero, F., Ackermann, M., Ajello, M., et al. 2015, ApJS, 218, 23, (3FGL) Adelman-McCarthy, J. K., Agüeros, M. A., Allam, S. S., et al. 2008, ApJS, 175,297

Atwood, W. B., Abdo, A. A., Ackermann, M., et al. 2009, ApJ, 697, 1071 Baldi, R. D., Capetti, A., \& Giovannini, G. 2015, A\&A, 576, A38 Barro, G., Faber, S. M., Pérez-González, P. G., et al. 2013, ApJ, 765, 104 Becker, R. H., White, R. L., \& Helfand, D. J. 1995, ApJ, 450, 559 Belfiore, F., Maiolino, R., Bundy, K., et al. 2015, MNRAS, 449, 867 Belfiore, F., Maiolino, R., Maraston, C., et al. 2016, MNRAS, 461, 3111 Belfiore, F., Maiolino, R., Maraston, C., et al. 2017, MNRAS, 466, 2570 Bell, E. F., Wolf, C., Meisenheimer, K., et al. 2004, ApJ, 608, 752 Benson, A. J., Bower, R. G., Frenk, C. S., et al. 2003, ApJ, 599, 38 Best, P. N., \& Heckman, T. M. 2012, MNRAS, 421, 1569

Best, P. N., Kaiser, C. R., Heckman, T. M., \& Kauffmann, G. 2006, MNRAS, 368, L67

Best, P. N., Kauffmann, G., Heckman, T. M., et al. 2005, MNRAS, 362, 25 Binette, L., Magris, C. G., Stasinka, G., \& Bruzual, A. G. 1994, A\&A, 292, 13 Binney, J., \& Tabor, G. 1995, MNRAS, 276, 663

Blanton, M. R., Bershady, M. A., Abolfathi, B., et al. 2017, AJ, 154, 28 Blanton, M. R., Hogg, D. W., Bahcall, N. A., et al. 2003, ApJ, 594, 186 Blanton, M. R., Schlegel, D. J., Strauss, M. A., et al. 2005, AJ, 129, 2562 Bower, R. G., Benson, A. J., Malbon, R., et al. 2006, MNRAS, 370, 645 Brown, M. J. I., Moustakas, J., Kennicutt, R. C., et al. 2017, ApJ, 847, 136 Bundy, K., Bershady, M. A., Law, D. R., et al. 2015, ApJ, 798, 7 Bundy, K., Ellis, R. S., Conselice, C. J., et al. 2006, ApJ, 651, 120 Buson, L. M., Sadler, E. M., Zeilinger, W. W., et al. 1993, A\&A, 280, 409 Calzetti, D. 2001, PASP, 113, 1449

Calzetti, D., Armus, L., Bohlin, R. C., et al. 2000, ApJ, 533, 682

Cappellari, M. 2008, MNRAS, 390, 71

Cappellari, M. 2017, MNRAS, 466, 798

Cappellari, M., \& Emsellem, E. 2004, PASP, 116, 138

Cattaneo, A., Faber, S. M., Binney, J., et al. 2009, Natur, 460, 213

Chabrier, G. 2003, PASP, 115, 763

Chang, Y.-Y., van der Wel, A., da Cunha, E., \& Rix, H.-W. 2015, ApJS, 219, 8

Chen, Y., Shi, Y., Tremonti, C. a., et al. 2016, NatCo, 7, 13269

Cheung, E., Bundy, K., Cappellari, M., et al. 2016, Natur, 533, 504

Cheung, E., Faber, S. M., Koo, D. C., et al. 2012, ApJ, 760, 131

Choi, J., Conroy, C., Moustakas, J., et al. 2014, ApJ, 792, 95

Cid Fernandes, R., Stasińska, G., Mateus, A., \& Vale Asari, N. 2011, MNRAS, 413, 1687

Ciotti, L., D'Ercole, A., Pellegrini, S., \& Renzini, A. 1991, ApJ, 376, 380

Ciotti, L., \& Ostriker, J. P. 1997, ApJL, 487, L105

Ciotti, L., \& Ostriker, J. P. 2001, ApJ, 551, 131

Ciotti, L., \& Ostriker, J. P. 2007, ApJ, 665, 1038

Ciotti, L., Ostriker, J. P., \& Proga, D. 2010, ApJ, 717, 708

Condon, J. J. 1984, ApJ, 287, 461

Condon, J. J., Cotton, W. D., Greisen, E. W., et al. 1998, AJ, 115, 1693

Conroy, C., Graves, G. J., \& van Dokkum, P. G. 2014, ApJ, 780, 33
Conroy, C., van Dokkum, P. G., \& Kravtsov, A. 2015, ApJ, 803, 77 Croton, D. J., Springel, V., White, S. D. M., et al. 2006, MNRAS, 365, 11 Demoulin-Ulrich, M.-H., Butcher, H. R., \& Boksenberg, A. 1984, ApJ, 285, 527

Drory, N., MacDonald, N., Bershady, M. A., et al. 2015, AJ, 149, 77 Dunlop, J. S., \& Peacock, J. A. 1990, MNRAS, 247, 19

Dunn, R. J. H., \& Fabian, A. C. 2006, MNRAS, 373, 959

Faber, S. M., Willmer, C. N. A., Wolf, C., et al. 2007, ApJ, 665, 265

Fabian, A. C. 1994, ARA\&A, 32, 277

Fabian, A. C. 2012, ARA\&A, 50, 455

Fabian, A. C., Sanders, J. S., Taylor, G. B., et al. 2006, MNRAS, 366, 417

Falcke, H., \& Biermann, P. L. 1999, A\&A, 342, 49

Falcón-Barroso, J., Sánchez-Blázquez, P., Vazdekis, A., et al. 2011, A\&A, 532, A95

Fang, J. J., Faber, S. M., Koo, D. C., \& Dekel, A. 2013, ApJ, 776, 63

Graham, M. T., Cappellari, M., Li, H., et al. 2018, MNRAS, 477, 4711

Graves, G. J., \& Schiavon, R. P. 2008, ApJS, 177, 446

Gunn, J. E., Siegmund, W. A., Mannery, E. J., et al. 2006, AJ, 131, 2332

Heckman, T. M., \& Best, P. N. 2014, ARA\&A, 52, 589

Hopkins, P. A., Hernquist, L., Cox, T. J., et al. 2005, ApJ, 630, 705

Ilbert, O., Salvato, M., Le Floc'h, E., et al. 2010, ApJ, 709, 644

Jiang, Y.-F., Ciotti, L., Ostriker, J. P., \& Spitkovsky, A. 2010, ApJ, 711, 125

Kauffmann, G., Heckman, T. M., White, S. D. M., et al. 2003, MNRAS, 341,33

Kennicutt, R. C., Jr., Hao, C.-N., Calzetti, D., et al. 2009, ApJ, 703, 1672

Kroupa, P., \& Weidner, C. 2003, ApJ, 598, 1076

Lagos, C. P., Crain, R. A., Schaye, Joop., et al. 2015, MNRAS, 452, 3815

Law, D. R., Cherinka, B., Yan, R., et al. 2016, AJ, 152, 83

Law, D. R., Yan, R., Bershady, M. A., et al. 2015, AJ, 150, 19

Lin, Y.-T., \& Mohr, J. J. 2007, ApJS, 170, 71

Maraschi, L., Ghisellini, G., \& Celotti, A. 1992, ApJL, 397, L5

Maraston, C., \& Strómbáck, G. 2011, MNRAS, 418, 2785

Martig, M., Bournaud, F., Teyssier, R., \& Dekel, A. 2009, ApJ, 707, 250

Martin, D. C., Fanson, J., Schiminovich, D., et al. 2005, ApJL, 619, L1

Mathews, W. G., \& Brighenti, F. 2003, ARA\&A, 41, 191

Matteo, T. D., Springel, V., \& Hernquist, L. 2005, Natur, 433, 604

McNamara, B. R., \& Nulsen, P. E. J. 2007, ARA\&A, 45, 117

Morganti, R. 2017, FrASS, 4, 42

Moustakas, J., Coil, A. L., Aird, J., et al. 2013, ApJ, 767, 50

Moutard, T., Arnouts, S., Ilbert, O., et al. 2016, A\&A, 590, 102

Nagar, N. M., Falcke, H., \& Wilson, A. S. 2005, A\&A, 435, 521

Narayan, R., Igumenshchev, I. V., \& Abramowicz, M. A. 2000, ApJ, 539, 798

Narayan, R., Yi, I., \& Mahadevan, R. 1995, Natur, 374, 623

Nyland, K., Young, L. M., Wrobel, J. M., et al. 2016, MNRAS, 458, 2221

O'Sullivan, E., Forbes, D. A., \& Ponman, T. J. 2001, MNRAS, 328, 461

Oke, J. B., \& Gunn, J. E. 1983, ApJ, 266, 713

Padmanabhan, N., Schlegel, D. J., Finkbeiner, D. P., et al. 2008, ApJ, 674, 1217

Rawlings, S., \& Saunders, R. 1991, Natur, 349, 138

Rawlings, S., Saunders, R., Eales, S. A., \& Mackay, C. D. 1989, MNRAS, 240,701

Salim, S., Charlot, S., Rich, R. M., et al. 2005, ApJL, 619, L39

Salim, S., Dickinson, M., Michael Rich, R., et al. 2009, ApJ, 700, 161

Salim, S., Rich, R. M., Charlot, S., et al. 2007, ApJS, 173, 267

Sarazin, C. L. 1986, RvMP, 58, 1

Sarzi, M., Falcón-Barroso, J., Davies, R. L., et al. 2006, MNRAS, 366, 1151

Sarzi, M., Shields, J. C., Schawinski, K., et al. 2010, MNRAS, 402, 2187

SDSS Collaboration, Albareti, F. D., Allende Prieto, C., et al. 2017, ApJS, 233, 25

Sikora, M., Begelman, M. C., \& Rees, M. J. 1994, ApJ, 421, 153

Simard, L., Mendel, J. T., Patton, D. R., et al. 2011, ApJS, 196, 11

Singh, R., van de Ven, G., Jahnke, K., et al. 2013, A\&A, 558, A43

Smee, S. A., Gunn, J. E., Uomoto, A., et al. 2013, AJ, 146, 32

Stoughton, C., Lupton, R. H., Bernardi, M., et al. 2002, AJ, 123, 485

Strateva, I., Ivezic, Z., Knapp, G. R., et al. 2001, AJ, 122, 1861

Tadhunter, C. 2016, A\&ARv, 24, 10

Thomas, D., Maraston, C., Bender, R., \& Mendes de Oliveira, C. 2005, ApJ, 621,673

Tinsley, B. M. 1979, ApJ, 229, 1046

Trager, S. C., Faber, S. M., Worthey, G., \& González, J. J. 2000, AJ, 120, 165

Vazdekis, A., Ricciardelli, E., Cenarro, A. J., et al. 2012, MNRAS, 424, 157

Wake, D. A., Bundy, K., Diamond-Stanic, A. M., et al. 2017, AJ, 154, 86

White, R. L., Becker, R. H., Helfand, D. J., \& Gregg, M. D. 1997, ApJ, 475,479

Willott, C. J., Rawlings, S., Blundell, K. M., \& Lacy, M. 1999, MNRAS, 309, 1017 
Worthey, G., Faber, S. M., \& Gonzalez, J. J. 1992, ApJ, 398, 69

Worthey, G., Tang, B., \& Serven, J. 2014, ApJ, 783, 20

Wright, E. L., Eisenhardt, P. R. M., Mainzer, A. K., et al. 2010, AJ, 140, 1868

Yan, L., Donoso, E., Tsai, C.-W., et al. 2013, AJ, 145, 55

Yan, R., \& Blanton, M. R. 2012, ApJ, 747, 61

Yan, R., Bundy, K., Law, D. R., et al. 2016, AJ, 152, 197
Yang, X., Mo, H. J., van den Bosch, F. C., et al. 2007, ApJ, 671, 153

York, D. G., Adelman, J., Anderson, J. E., Jr., et al. 2000, AJ, 120, 1579

Young, L. M., Bureau, M., Davis, T. A., et al. 2011, MNRAS, 414, 940

Young, L. M., Scott, N., Serra, P., et al. 2014, MNRAS, 444, 3408

Yuan, F., \& Narayan, R. 2014, ARA\&A, 52, 529

Zensus, J. A. 1997, ARA\&A, 35, 607 